\title{
Routing a Mix of Conventional, Plug-in Hybrid, and Electric Vehicles
}

\author{
Gerhard Hiermann ${ }^{\mathrm{a}, *}$, Richard F. Hartl ${ }^{\mathrm{a}}$, Jakob Puchinger ${ }^{\mathrm{b}, \mathrm{c}}$, Thibaut Vidal ${ }^{\mathrm{d}}$ \\ ${ }^{a}$ Department of Business Administration, University of Vienna, Austria \\ ${ }^{b}$ Laboratoire Genie Industriel, CentraleSupélec, Université Paris-Saclay, France \\ ${ }^{c}$ Institut de Recherche Technologique SystemX, Palaiseau, France \\ ${ }^{d}$ PUC-Rio, Pontifical Catholic University of Rio de Janeiro, Brazil
}

\begin{abstract}
We introduce an electric vehicle routing problem combining conventional, plug-in hybrid, and electric vehicles. Electric vehicles are constrained in their service range by their battery capacity, and may require time-consuming recharging operations at some specific locations. Plug-in hybrid vehicles have two engines, an internal combustion engine and an electric engine using a built-in rechargeable battery. These vehicles can avoid visits to recharging stations by switching to fossil fuel. However, this flexibility comes at the price of a generally higher consumption rate and utility cost.

To solve this complex problem variant, we design a sophisticated metaheuristic which combines a genetic algorithm with local and large neighborhood search. All route evaluations, within the approach, are based on a layered optimization algorithm which combines labeling techniques and greedy evaluation policies to insert recharging stations visits in a fixed trip and select the fuel types. The metaheuristic is finally hybridized with an integer programming solver, over a set partitioning formulation, so as to recombine high-quality routes from the search history into better solutions. Extensive experimental analyses are conducted, highlighting the good performance of the algorithm and the contribution of each of its main components. Finally, we investigate the impact of fuel and energy cost on fleet composition decisions. Our experiments show that a careful use of a mixed fleet can significantly reduce operational costs in a large variety of price scenarios, in comparison with the use of a fleet composed of a single vehicle class.
\end{abstract}

Keywords: Routing, Electric Vehicles, Plug-In Hybrid, Heterogeneous Fleets, Hybrid Metaheuristics

\section{Introduction}

Climate change is a major concern for humankind, and it is essential to reduce anthropogenic greenhouse gas emissions. Major emission sources are energy production, industry, agriculture, and transportation. Governmental institutions all over the world seek to reduce the emission of carbon-dioxide equivalents by introducing new regulations (Edenhofer et al., 2014). This has led to increased research into more efficient and cleaner ways to use fossil fuel as well as alternative types of engines. In recent years, the production of battery electric vehicles (BEVs) for large markets has increased, and considerable efforts have been made to reduce vehicle and battery costs (AustriaTech, 2014). However, the relatively limited capacity of batteries considerably reduces the operational range of BEVs, such that time-consuming visits to recharging stations must be considered in the planning phase.

Hybrid vehicles with features of both BEVs and internal combustion engine vehicles (ICEVs) have been developed to reduce the necessary stops and the infrastructure dependence. The so-called plug-in hybrid electric vehicles (PHEVs) have two engines - an internal combustion engine (ICE) and a pure electric enginethat can be easily switched, permitting the use of electric mode on selected route segments. PHEVs do not

\footnotetext{
${ }^{*}$ Corresponding author

Email addresses: ghiermann@hotmail.com (Gerhard Hiermann), richard.hartl@univie.ac.at (Richard F. Hartl), jakob.puchinger@centralesupelec.fr (Jakob Puchinger), vidalt@inf.puc-rio.br (Thibaut Vidal)
} 
have the operational range restriction of BEVs, and they can recharge en route to reduce the use of fossil fuel on other trip segments, which can be beneficial in terms of cost and emissions. However, two engines also mean a heavier base load, which in turn lead to a higher consumption of both electricity and fuel. Each technology has its merits, and with the variety of subtypes available (AustriaTech, 2014), choosing the best fleet mix for a given transport demand is a very difficult task.

In this article, we introduce a hybrid heterogeneous electric fleet routing problem with time windows and recharging stations $\left(\mathrm{H}^{2} \mathrm{E}-\mathrm{FTW}\right)$. This problem considers three different vehicle classes-ICEV, BEV, and PHEV - as well as multiple vehicle types for each class, differing in capacity, battery size, and electric energy and/or fuel consumption per mile. The batteries of BEVs and PHEVs can be charged at recharging stations. To retain a simple problem formulation and focus on the fleet-composition decisions, we assume that the recharging time is proportional to the amount of energy charged. For PHEVs, the engine type used can be switched at any given time by the driver or an on-board unit. The routing cost consists of the electric energy and the fuel consumption weighted by their respective costs. The number of vehicle classes and types used in the final mix is not limited, but a fixed cost per usage balances the fleet cost with the consumption-based variable cost. By integrating tour planning and vehicle selection within this problem, we aim to achieve better overall plans compared to those obtained by sequential decisions. To this date, integrated approaches have not been often considered in the literature, especially for ICEVs, BEVs, and PHEVs of different types.

The three vehicle classes differ in the need to recharge and to choose the engine. To account for these decisions, we propose a systematic route evaluation approach using decision layers, which separate the vehiclespecific knowledge from the higher-level routing optimization procedure. This generic design allows us to apply state-of-the-art metaheuristic approaches and operators. During the search, each route is evaluated using a labeling algorithm for the placement of recharging stations with a greedy policy for engine-mode choices and resource-consumptions computations. Finally, we use an efficient hybrid genetic algorithm (HGA) for routing optimization. This method combines a local search (LS) for solution intensification, state-of-the-art population-diversity management techniques, a set partitioning component which creates new solutions based on routes discovered in the past search, and a large neighborhood search (LNS) which acts as a mutation operator.

Through extensive numerical studies, we evaluate the performance of this metaheuristic on a variety of benchmark instances and problem variants. For the special case of the heterogeneous fleet size and mix problem considering only BEVs (E-FSMFTW; Hiermann et al., 2016; Montoya, 2016), our approach finds solutions of better quality than previous algorithms in a similar time. Additional comparative analyses, on the electric VRPTW with recharging stations (E-VRPTW; Schneider et al., 2014) and partial recharging (E-VRPTWPR; Keskin \& Çatay, 2016) further demonstrate the competitiveness of our approach. Finally, to gain additional managerial insights, we performed sensitivity analysis to evaluate the merits of a mixed vehicle fleet, and measure the impact of fuel and electricity costs on fleet composition. The main contributions of this work are:

- the definition of an integrated routing and vehicle selection problem with ICEVs, PHEVs, and BEVs;

- a sophisticated solution algorithm, based on a new systematic search method using multiple decision layers for route evaluation;

- an extension of the metaheuristic with a LNS and set partitioning component;

- finally, a detailed sensitivity analysis on the impact of mixed fleet optimization and different vehicle types on operational costs, for multiple fuel and electricity cost scenarios.

\section{Problem statement}

In the Hybrid Heterogeneous Electric Fleet routing problem with Time Windows and recharging stations $\left(\mathrm{H}^{2} \mathrm{E}-\mathrm{FTW}\right)$, we are given a graph $\mathcal{G}(\mathcal{V} \mathcal{E})$ and a set of vehicle types $\mathcal{K}=\mathcal{K}^{I} \cup \mathcal{K}^{B} \cup \mathcal{K}^{P}$, which is the union of the vehicles in the three classes: $\operatorname{ICEV}\left(\mathcal{K}^{I}\right), \operatorname{BEV}\left(\mathcal{K}^{B}\right)$, and $\operatorname{PHEV}\left(\mathcal{K}^{P}\right)$. The set of vertices $\mathcal{V}=\left\{v_{0}, \ldots, v_{n+p}\right\}$ consists of a single depot $v_{0}$, a set of $n$ customers $\mathcal{C}=\left\{v_{1}, \ldots, v_{n}\right\}$, and a set of $p$ recharging stations $\mathcal{F}=\left\{v_{n+1}, \ldots, v_{n+p}\right\}$. Each tour starts and ends at the depot $v_{0}$, and each customer is visited exactly once. Recharging stations are optional and can be visited by any vehicle multiple times or not at all. 
A demand $q_{i}$ and service time $s_{i}$ is associated to each customer $v_{i} \in \mathcal{V}$, and each vehicle $k$ has a capacity $Q^{k}$. For each vertex $v_{i} \in \mathcal{V}$, we define a service time window $\left[e_{i}, l_{i}\right]$, where $e_{i}$ is the earliest and $l_{i}$ the latest possible start of service. Recharging stations can be used to recharge the batteries between customer visits. Each vehicle type with a battery (i.e., BEV or PHEV) can recover a quantity of energy between the current charge level and the battery capacity $Y^{k}, k \in \mathcal{K}^{B} \cup \mathcal{K}^{P}$. Recharging takes a time which is assumed to be linear in the quantity of energy recharged with an inverse recharging rate $g$, (i.e., time $=$ amount $\cdot g$ ). This implies that the decision on how much energy to charge is part of the routing problem. The vehicles are assumed to be fully charged when leaving the depot, and the charge level is not allowed to drop below zero. Replenishment operations for conventional engines (i.e., ICEV or PHEV) are usually fast, and therefore can be ignored in the problem.

Each vehicle $k$ has an associated fixed cost, denoted $f^{k}$, which may represent some fraction of the acquisition, maintenance, and driver cost. The electric energy and fuel consumption rates are constants, denoted $r_{\mathrm{E}}$ and $r_{\mathrm{F}}$ respectively. Since ICEVs cannot use electricity to travel between vertices, $r_{\mathrm{E}}$ is defined only for $k \in \mathcal{K}^{B} \cup \mathcal{K}^{P}$. The same holds for fuel, i.e., $r_{\mathrm{F}}$ is defined only for $k \in \mathcal{K}^{I} \cup \mathcal{K}^{P}$. Vehicles traverse the graph using edges $(i, j) \in \mathcal{E}, i, j \in \mathcal{V}$, where $\mathcal{E}$ satisfies the triangle inequality. Each edge has two associated values, the distance $d_{i j}$ and travel time $t_{i j}$. The electric energy and fuel consumption are linear functions of the distance and the corresponding consumption rate, i.e., $d_{i j} \cdot r_{\mathrm{E}}$ and $d_{i j} \cdot r_{\mathrm{F}}$ respectively. Both values can be calculated in advance for each edge and each applicable vehicle type.

PHEVs include an electric engine and an ICE. We assume that the driver can switch between them without any restrictions or penalties other than the current charge level, possibly on the way between two vertices. Finally, an electricity price $c_{\mathrm{E}}$ and a fuel price $c_{\mathrm{F}}$ are defined. These constant prices should be multiplied by the total electric energy and fuel consumed by the fleet. The objective of the $\mathrm{H}^{2} \mathrm{E}-\mathrm{FTW}$ is to minimize the sum of the fixed and variable costs.

\section{Related work}

The literature on vehicle routing problems (VRP) is rich and diverse. Problems with different vehicle types are usually called heterogeneous VRPs, and are reviewed in Baldacci et al. (2008) and Toth \& Vigo (2014). The research on electric vehicles has progressed on various aspects: battery technology, optimal routing, and tour planning. We refer to Pelletier et al. (2016) and Juan et al. (2016) for a recent survey of this broad research field. We now provide a short overview of directly related work.

Conrad \& Figliozzi (2011) presented one of the earliest studies of electric vehicle routing, where electric vehicles can be fast-charged to $80 \%$ of the maximum battery level at customer locations. Called the "recharging VRP", this formulation assumed a fixed charging time independent of the battery state but contributing to the objective value by imposing a recharging cost. Erdoğan \& Miller-Hooks (2012) formulated a green VRP using alternative-fuel vehicles that can be refueled at dedicated stations. Electric vehicle routing and recharging stations were first considered by Schneider et al. (2014), who introduced the E-VRPTW. Desaulniers et al. (2016) proposed a branch-and-cut-and-price algorithm for this problem, with a bidirectional labeling algorithm for full and partial recharging at single or multiple recharging stations. Moreover, Keskin \& Çatay (2016) and Çatay \& Keskin (2017) studied problem variants with possible partial recharging and quick recharging stations, respectively.

Felipe et al. (2014) studied multiple recharging technologies with different costs for a single fleet type. With regards to fleet mix, Hiermann et al. (2016) extended the E-VRPTW by considering a heterogeneous fleet of BEVs. Goeke \& Schneider (2015) tackled a variant of the E-VRPTW with a single type of ICEV and BEV with load-dependent energy consumption. Lebeau et al. (2015) considered multiple types of ICEVs and BEVs and proposed a small benchmark set of 21 instances, using distances from a real-world road network. However, in their formulation, recharging is possible only at the depot. A rich formulation is studied by Sassi et al. (2015). The authors consider a combined fleet and synchronization aspects of the recharging grid. A regret-based construction heuristic is initially used to solve large real-world instances.

This research focuses on the tour planning, but there have also been studies of street routing problems with electric vehicles. In particular, Zündorf (2014) provides methods to incorporate different charging technologies and presents an algorithm to determine a set of shortest energy-efficient paths. Finally, for 
PHEVs the literature is rather sparse, with Abdallah (2013) being one of the first to discuss a VRPTW using a homogeneous fleet of PHEVs. The problem definition allows the vehicle to be recharged before or after serving a customer at the customer's location.

\section{Methodology - Route Evaluations}

The problem variant presented in this work is quite complex: even the evaluation of a single route (sequence of customer visits) can be a hard task due to the necessity of optimizing visits to recharging stations, arrival times, and possible engine mode changes. Based on this problem decomposition, we design a heuristic algorithm that works on solutions represented as sequences of customer visits without charging stations, with their associated vehicle types. Whenever a route needs to be evaluated, the method finds routing cost by means of labeling techniques for charging-stations insertions, in combination with greedy policies for charging-levels and fuel-selection decisions. The remainder of this section details the route evaluation techniques, whereas Section 5 describes the routing search heuristic built on top of this evaluation method.

The $\mathrm{H}^{2} \mathrm{E}-\mathrm{FTW}$ includes three vehicle classes. ICEV routing is related to the classical VRPTW (Toth \& Vigo, 2014), in which no energy restrictions have to be considered. The BEVs have additional energy constraints. BEV routing is related to the E-VRPTW (Schneider et al., 2014), with possible partial recharging, i.e., the amount of recharging is a decision variable, as in Keskin \& Çatay (2016). PHEVs further extend the E-VRPTW - referred to as the PH-VRPTW in this work - by adding decisions about on the propulsion mode used. Table 4.1 summarizes the features of each subproblem.

\begin{tabular}{r|c|c|c}
\hline Feature & ICEV (VRPTW) & BEV (E-VRPTW) & PHEV (PH-VRPTW) \\
\hline Itinerary & $\mathrm{x}$ & $\mathrm{x}$ & $\mathrm{x}$ \\
Demand & $\mathrm{x}$ & $\mathrm{x}$ & $\mathrm{x}$ \\
Time windows & $\mathrm{x}$ & $\mathrm{x}$ & $\mathrm{x}$ \\
Energy & & $\mathrm{x}$ & $\mathrm{x}$ \\
(Partial) recharging & & $\mathrm{x}$ & $\mathrm{x}$ \\
Propulsion mode & & & $\mathrm{x}$ \\
\hline
\end{tabular}

Table 4.1: Comparison of features of each subproblem

Each evaluation of a route, represented as a simple sequence of customer visits, requires to solve a subproblem to insert visits to recharging stations, determine arrival times and possible engine mode changes, depending on the vehicle type. Even if this strategy appears at first glance to lead to a higher computational effort, it allows to apply state-of-the-art metaheuristic search strategies on a simpler abstraction of the problem as a VRP, relegating vehicle specificities to the route evaluation operators. Many methods in the literature implicitly use this concept; e.g., when solving time-constrained VRP variants, it is common to optimize customers assignments to vehicles and visit sequences using metaheuristic search, whereas customers visit times and costs are determined by solving a timing subproblem for each route (Vidal et al., 2015b).

Figure 4.1 describes the general organization of the method. The top search layer includes a state-of-theart HGA. It optimizes the assignment of customers to vehicles and the sequences of visits. Each sequence of visits enumerated by the HGA must be evaluated. For that purpose, the first route-evaluation layer of the E/PH-VRPTW solves a resource-constrained shortest path problem (RCSPP) using dynamic programming (DP) to determine where to insert recharging stations. Moreover, to evaluate the cost of each sub-path in the labeling algorithm, a greedy policy is called. This component will be referred as the "second" evaluation layer.

The following sections provide a bottom-to-top description of the route-evaluation layers for the EVRPTW and PH-VRPTW, starting from the greedy evaluation policy (second layer) which evaluates the cost of a sequence of visits to customers and recharging stations, and pursuing with the labeling algorithm (first layer) in charge of charging-stations insertions. Note that we use the term "energy" to refer to electric energy only. 


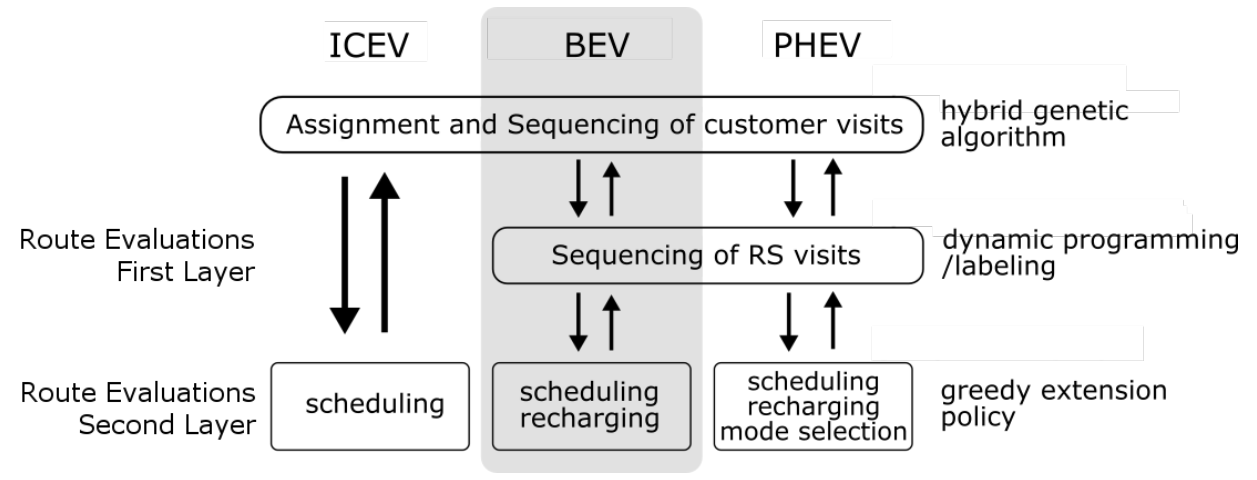

Figure 4.1: General structure of the solution method

\subsection{Second layer of the route evaluations: Greedy extension policies}

The second route-evaluation layer considers a known sequence of visits to customers and recharging stations and seeks to optimize partial-recharging levels, engine-mode choices when applicable and visit times. We use the notation of resource extension functions (REF) to define how the value of a resource (e.g., distance) changes when extending a sequence with an additional vertex (Irnich \& Villeneuve, 2006).

\section{Greedy charging policy for BEV}

The extension functions for the E-VRPTW with complete recharging have been previously introduced in Hiermann et al. (2016). However, these functions must be adapted to consider partial recharging. The amount of recharging must be determined for every recharging station visit. Since charging operations take time, every decision has a direct impact on the feasibility of a route (e.g., the time windows might be violated). To solve this problem, we apply a greedy recharging policy with two simple rules:

1. Whenever a sequence ending in vertex $i$ is extended to vertex $j$, and the electric energy required $\left(d_{i j} \cdot r_{\mathrm{E}}\right)$ exceeds the current state, attempt to recharge the required amount at the last recharging station visited.

2. Whenever a sequence is extended to a customer $j$ and the arrival time is prior to the earliest begin of service time, use the waiting time to perform a recharging operation at the last station visited.

This policy exploits three properties of the problem:

- a vehicle needs to recharge only if it cannot complete the tour otherwise;

- electricity costs are applied to the amount consumed, not to the amount recharged;

- recharging operations can always be performed at the last recharging station visited.

The first property is straightforward: we recharge only if really necessary. The second states that the amount of energy recharged has no impact on the routing costs; this implies that waiting time can always be transformed into a charging operation. The third property exploits the linear recharging rate. A linear rate implies that all time-feasible choices of the location of the recharging operation are equivalent for all visits to recharging stations in the sequence so far, as long as they fulfill the energy capacity constraint. Therefore, using only the last one will not reduce the quality of the recharging decision.

This policy is captured by defining the following functions to extend the resources processed for a given visit sequence $\{d, \ldots, i\}$ to a new visit $j$. Due to the number of variables involved, we describe intermediate calculations (starting with $\Delta$ ) only briefly in the text where they occur to simplify the descriptions. At the end of the section, these intermediate calculations will be detailed and commented.

$$
\begin{aligned}
T_{j}^{\mathrm{COST}} & =T_{i}^{\mathrm{COST}}+d_{i j} \cdot r_{\mathrm{E}} \cdot c_{\mathrm{E}} \\
T_{j}^{\mathrm{Q}} & =T_{i}^{\mathrm{Q}}+q_{j}
\end{aligned}
$$

The cost and capacity resources are $T_{i}^{\mathrm{COST}}$ and $T_{i}^{\mathrm{Q}}$ respectively. An extension simply sums the cost of the 
consumed electric energy and additional load respectively.

$$
\begin{aligned}
T_{j}^{\mathrm{DUR}} & =T_{i}^{\mathrm{DUR}}+t_{i j}+s_{j}+\Delta^{\mathrm{WT}}+\Delta^{\mathrm{T}^{\mathrm{RC}}} \\
T_{j}^{\mathrm{TW}} & =T_{i}^{\mathrm{TW}}+\Delta^{\mathrm{TW}}+\Delta^{\mathrm{TW}}
\end{aligned}
$$

The time resources are $T_{i}^{\mathrm{DUR}}$ for the duration of the route and $T_{i}^{\mathrm{TW}}$ for the time warp used. Using the concept of a time warp allows for a more efficient penalty scheme (Nagata et al., 2010; Vidal et al., 2015a) because time-window violations are accounted for and repaired locally and do not propagate any further. The duration is calculated by adding the travel time $t_{i j}$, the service time $s_{j}$, the waiting time $\Delta^{\text {wT }}$, and the recharging time $\Delta^{\mathrm{T}}$. The time warp $T_{i}^{\mathrm{TW}}$ is extended by adding any necessary time warp as a result of either arriving too late at the customer $\left(\Delta^{\mathrm{TW}}\right)$ or due to a battery recharging operation $\left(\Delta^{\mathrm{TW}}\right)$.

$$
\begin{aligned}
T_{j}^{\mathrm{Y}} & =T_{i}^{\mathrm{Y}}-d_{i j} \cdot r_{\mathrm{E}}+\Delta^{\mathrm{Y}^{\mathrm{RC}}}+\Delta^{\mathrm{Y}^{\mathrm{WT}}} \\
T_{j}^{\mathrm{EY}} & =T_{i}^{\mathrm{EY}}+\Delta^{\mathrm{EY}}
\end{aligned}
$$

$T_{i}^{\mathrm{Y}}$ is the available electric energy of the vehicle at vertex $i$, whereas $T_{i}^{\mathrm{EY}}$ counts the energy constraint violations. $T_{j}^{\mathrm{Y}}$ is calculated by subtracting the electric energy required to reach $j\left(\mathrm{i} . e ., d_{i j} \cdot r_{\mathrm{E}}\right.$ ) and adding the energy recharged to reach node $\mathrm{j}\left(\Delta^{\mathrm{Y}^{\mathrm{RC}}}\right)$, as well as the amount recharged to avoid waiting time at the customer $\left(\Delta^{\mathrm{Y}^{\mathrm{WT}}}\right)$. Two additional resources must be processed: the amount of electricity that can be recharged $T_{i}^{\mathrm{YAR}}$ and the time available for recharging $T_{i}^{\mathrm{TAR}}$. First,

$$
T_{j}^{\mathrm{YAR}}= \begin{cases}Y-\left(T_{i}^{\mathrm{Y}}-d_{i j} \cdot r_{\mathrm{E}}+\Delta^{\mathrm{YC}}+\Delta^{\mathrm{Y}^{\mathrm{WT}}}\right) & \text { if } j \in \mathcal{F}, \\ T_{i}^{\mathrm{YAR}}-\left(\Delta^{\mathrm{Y}^{\mathrm{RC}}}-\Delta^{\mathrm{EY}}+\Delta^{\mathrm{Y}}\right) & \text { otherwise. }\end{cases}
$$

$T_{i}^{\mathrm{YAR}}$ is the amount of electric energy that can be recharged at the last recharging station visited. It ensures that the maximum battery capacity is respected. If $j$ is a recharging station, then the rechargeable amount is the difference between the maximum capacity $Y$ and the current capacity of the battery (4.5). Otherwise, if $j$ is a customer or the depot, the extended resource incorporates the amount of energy actually recharged in order to reach $j$ (i.e., $\Delta^{\mathrm{Y}^{\mathrm{RC}}}-\Delta^{\mathrm{EY}}$ ) or used because of waiting time $\left(\Delta^{\mathrm{YC}}\right)$. Second,

$$
T_{j}^{\mathrm{TAR}}= \begin{cases}\max \left\{0, l_{j}-\left(e_{0}+\Delta\right)\right\} & \text { if } j \in \mathcal{F}, \\ \max \left\{0, \min \left\{T_{i}^{\mathrm{TAR}}-\Delta^{\mathrm{T}^{\mathrm{RC}}}-\Delta^{\mathrm{Y}^{\mathrm{wT}}} \cdot g, l_{j}-\left(e_{0}+\Delta+\Delta^{\mathrm{WT}}\right)\right\}\right\} & \text { otherwise. }\end{cases}
$$

$T_{i}^{\mathrm{TAR}}$ ensures that the required time warp due to recharging operations is identified correctly. If $j \in \mathcal{F}$, the available recharging time is reset to the time remaining until the end of the time window. Otherwise, the minimum of two cases defines the extension: 1) the previous available time minus the time required to recharge in order to reach vertex $j$ (i.e., $\left.\Delta^{\mathrm{T}^{\mathrm{RC}}}\right)$ and due to waiting time $\left(\Delta^{\mathrm{Y}^{\mathrm{WT}}} \cdot g\right.$, where $g$ is the inverse recharging rate), and 2) the remaining time window of the current customer. 
Finally, the intermediate calculations are displayed below:

$$
\begin{aligned}
& \Delta^{\mathrm{Y}^{\mathrm{RC}}}=\max \left\{d_{i j} \cdot r_{\mathrm{E}}-T_{i}^{\mathrm{Y}}, 0\right\} \\
& \Delta^{\mathrm{EY}}=\max \left\{\Delta^{\mathrm{RC}}-T_{i}^{\mathrm{YAR}}, 0\right\} \\
& \Delta^{\mathrm{T}^{\mathrm{RC}}}=\Delta^{\mathrm{Y}^{\mathrm{RC}}} \cdot g \\
& \Delta^{\mathrm{TW}^{\mathrm{RC}}}=\max \left\{\Delta^{\mathrm{T}^{\mathrm{RC}}}-T_{i}^{\mathrm{TAR}}, 0\right\} \\
& \Delta=T_{i}^{\mathrm{DUR}}-T_{i}^{\mathrm{TW}}+\Delta^{\mathrm{T}^{\mathrm{RC}}}-\Delta^{\mathrm{TW}}+t_{i j} \\
& \Delta^{\mathrm{WT}}=\max \left\{e_{j}-\Delta-e_{0}, 0\right\} \\
& \Delta^{\mathrm{TW}}=\max \left\{e_{0}+\Delta-l_{j}, 0\right\} \\
& \Delta^{\mathrm{Y}^{\mathrm{WT}}}=\min \left\{T_{i}^{\mathrm{YAR}}-\Delta^{\mathrm{Y}^{\mathrm{RC}}}, \min \left\{T_{i}^{\mathrm{TAR}}-\Delta^{\mathrm{T}^{\mathrm{RC}}}, \Delta^{\mathrm{WT}}\right\} / g\right\}
\end{aligned}
$$

Equation (4.9) calculates the energy required to reach vertex $j$ that is not covered by the energy stored in the battery $\left(T_{i}^{\mathrm{Y}}\right)$. The amount that is not rechargeable is calculated in (4.10) and depends on the charge available at the last recharging station visited $\left(T_{i}^{\mathrm{YAR}}\right)$. Equation (4.11) determines the time required for recharging the missing amount, and (4.12) stores the time warp required to satisfy the time window constraint. After this step, the actual duration is calculated using (4.13), which determines the waiting time and time warp occurring in vertex $j$. Equations (4.14) and (4.15) calculate the waiting time at vertex $j$ and the time warp required, respectively. The second rule of our policy is enforced by (4.16), which determines the amount of recharging that can replace waiting at vertex $j$.

\section{Greedy charging and mode selection policy for PHEV}

PHEVs pose an additional challenge because the availability of two engines introduces the need to select the propulsion mode. We assume that the mode can be changed at any time during the tour at no additional cost. Furthermore, the battery can be recharged in the same way as that of BEVs.

As for the BEV case, a sequence of customer and recharging stations visits is received as input of the greedy algorithm. Our evaluation uses the following rules to select the propulsion mode and to guarantee feasibility in time and energy, if possible:

1. Use electric energy mode in priority;

2. When a sequence ending in vertex $i$ is extended to vertex $j$, and the energy required $d_{i j} \cdot r_{\mathrm{E}}$ exceeds the current state, try to recharge the required amount at the last recharging station visited;

3. Avoid waiting time by recharging;

4. When a time window is violated, exchange the time spent recharging for the corresponding amount of fuel. The first item comes directly from the assumption that electricity is cheaper than fossil fuel. Otherwise, no electricity would be required, reducing the problem to the variant with no energy constraints. The second and third points are identical to the BEV policy described above, ensuring that energy is recharged only when necessary or to avoid waiting time. In the BEV case, a time window can be violated because the vehicle arrives too late or charging is required. In contrast, a PHEV can use the ICE to save recharging time. This is handled by the last item of the policy. Of course, only recharging time can be reverted to avoid a time warp. A detailed description analogous to the BEV case can be found in the Appendix.

\subsection{First layer of the route evaluations: Insertion of recharging station visits}

The planning of recharging-station visits is performed by a labeling algorithm, relying on the second-layer greedy policies to calculate the cost and other resources of each sub-path. Given a fixed sequence of visits, the labeling algorithm aims to determine which recharging station, if any, should be used in the sequence. This problem can generally be formulated as a RCSPP (Irnich \& Desaulniers, 2005).

Let $\pi=\left\{v_{0}, v_{1}, v_{2}, \ldots, v_{0}\right\}$ be an itinerary without any recharging stations. The goal is to find the best recharging stations in $\mathcal{F}$ and their placement in $\pi$ to obtain an energy and time feasible, cost minimal route with possible recharging stations. The problem is transformable to the fixed-sequence arc selection problem 


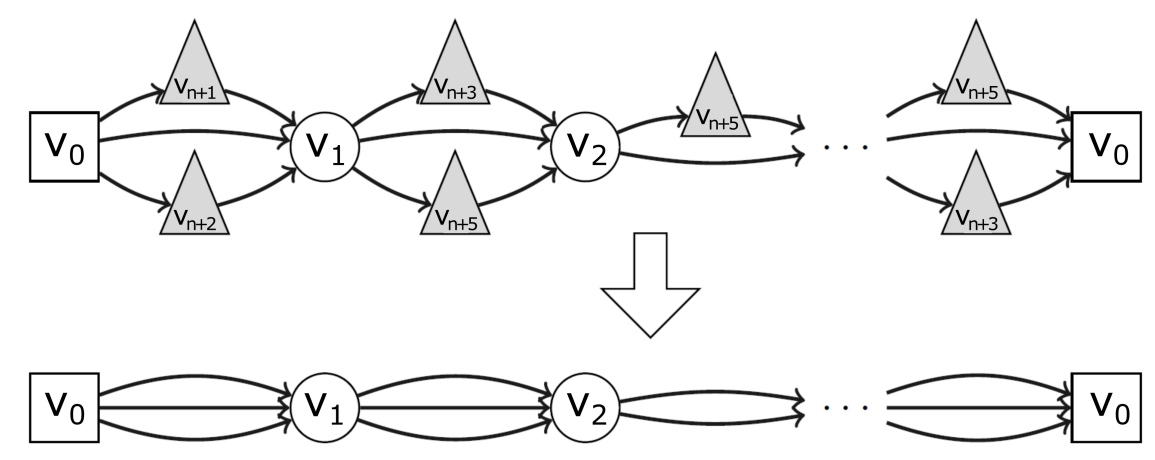

Figure 4.2: Transformation of the problem of finding the best path with recharging station visits to a fixed-sequence arc selection problem.

described in Garaix et al. (2010) by representing each possible detour to a recharging station as a parallel arc in a directed acyclic multigraph; see Figure 4.2.

To solve this problem, we use a labeling algorithm following the general steps of Irnich \& Desaulniers (2005) but differing in the label definition, the extension, and the dominance criterion. The description of the labeling algorithm uses a function-style notation to specify the value of a field of a label. The resources are defined specifically for each problem to ensure efficient handling of the dominance checks.

The resources for the BEV labeling are as follows:

$$
R^{\mathrm{BEV}}=\left\{v, T^{\mathrm{COST}}, T^{\mathrm{DUR}}, T^{\mathrm{TW}}, T^{\mathrm{Y}}, T^{\mathrm{YAR}}, T^{\mathrm{TAR}}\right\}
$$

where $T^{\operatorname{CosT}}(L)$ is the cost of label $L, T^{\mathrm{DUR}}(L)$ the time duration at $v(L)$, the timewarp $T^{\mathrm{TW}}(L)$, and $T^{\mathrm{Y}}(L)$ the current energy level. $T^{\mathrm{YAR}}(L)$ is the rechargeable energy and $T^{\mathrm{TAR}}(L)$ the maximum recharging time, both at the last recharging station visited. These values are directly obtained from the greedy evaluation described in Section 4.1.

The dominance criterion is defined as follows: A label $L_{1}$ dominates $L_{2}$ if its has a lower sum of cost and timewarp penalty (4.17), a shorter duration (4.18), and a higher maximal available energy (4.19), i.e., the current level plus the rechargeable amount. Moreover, the available electric energy must either be higher, or the total duration must be shorter after recharging the missing amount, as described in Equation (4.20).

$$
\begin{aligned}
T^{\mathrm{COST}}\left(L_{1}\right)+\rho^{\mathrm{TW}} \cdot T^{\mathrm{TW}}\left(L_{1}\right) & \leq T^{\mathrm{COST}}\left(L_{2}\right)+\rho^{\mathrm{TW}} \cdot T^{\mathrm{TW}}\left(L_{2}\right) \\
T^{\mathrm{DUR}}\left(L_{1}\right) & \leq T^{\mathrm{DUR}}\left(L_{2}\right) \\
T^{\mathrm{Y}}\left(L_{1}\right)+\min \left\{T^{\mathrm{YAR}}\left(L_{1}\right), T^{\mathrm{TAR}}\left(L_{1}\right) / g\right\} & \geq T^{\mathrm{Y}}\left(L_{2}\right)+\min \left\{T^{\mathrm{YAR}}\left(L_{2}\right), T^{\mathrm{TAR}}\left(L_{2}\right) / g\right\} \\
\left(T^{\mathrm{Y}}\left(L_{1}\right) \geq T^{\mathrm{Y}}\left(L_{2}\right)\right) \vee\left(T^{\mathrm{DUR}}\left(L_{1}\right)\right. & \left.+\left(T^{\mathrm{Y}}\left(L_{2}\right)-T^{\mathrm{Y}}\left(L_{1}\right)\right) \cdot g \leq T^{\mathrm{DUR}}\left(L_{2}\right)\right)
\end{aligned}
$$

where $\rho^{\mathrm{TW}}$ is the penalty weight for time window violations, which will be described in the following section in detail.

For the case of PHEV, the labeling algorithm incorporates additional variables and dominance rules, described in the Appendix.

\section{Methodology - Metaheuristic Search}

Finally, to obtain good assignment and routing decisions, we use a metaheuristic approach based on the HGA introduced in Vidal et al. (2012, 2013). Our adaptation of the method includes two additional components: a set partitioning component to recombine routes encountered during the search process, and a destroy-and-recreate component, which acts as a fast mutation operator.

The general structure of the algorithm is shown in Figure 5.1. Two populations are maintained during the search, containing the feasible and infeasible individuals, respectively. New individuals, called offspring, are 


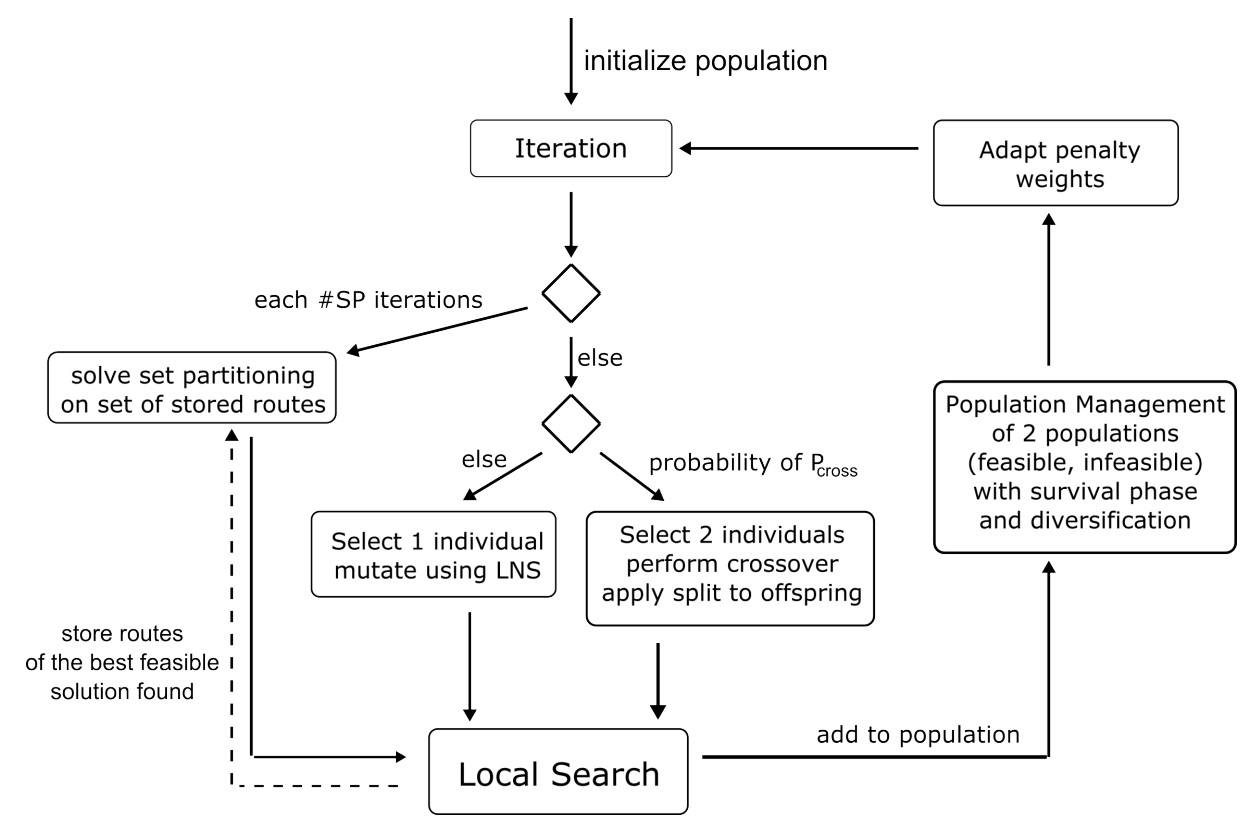

Figure 5.1: General overview of the HGA developed in this paper.

generated at each iteration by recombination, mutation or set partitioning. The set partitioning component is called every $i t^{\mathrm{SP}}$ iterations, combining the routes found during the search. At every other iteration, a recombination or mutation is performed with probability $p^{\mathrm{CROSS}}$ and $1-p^{\mathrm{CROSS}}$, respectively, so as to generate a single offspring which is improved by LS and included in the appropriate population. Based on the size of the population and the performance of the search, survival and diversification mechanisms are triggered.

The remainder of this section details each component of the HGA. Section 5.1 discusses the representation of a solution and the evaluation of individuals. Section 5.2 explains parent selection, recombination, and mutation. Section 5.3 presents the LS procedure, and Section 5.4 discusses the population management.

\subsection{Solution representation and evaluation}

A solution $s$ is represented as a set of routes and vehicle types. Each route starts at the depot, visits customer vertices, and ends at the depot. Recharging stations are only considering during route evaluations, as described in Section 4.1.

Let $R(s)$ represent the routes in $s$, and let $T_{r}$ be the computed evaluation data of each route $r \in R(s)$. A feasible solution does not allow any time window, energy, or capacity violations, i.e.,

$$
\left(T_{r}^{\mathrm{Q}} \leq Q^{r}\right) \wedge\left(T_{r}^{\mathrm{EY}} \leq 0\right) \wedge\left(T_{r}^{\mathrm{TW}} \leq 0\right)
$$

where $Q^{r}$ is the maximal load capacity of the vehicle assigned to route $r$. Infeasible solutions are obtained by relaxing the vehicle capacities and time-window constraints. Constraint violations are penalized using weights which are adapted during the search. The objective value of a route $r$ is defined as the combination of the general routing cost $T_{r}^{\operatorname{COST}}$ and the weighted sum of all violations, i.e.,

$$
\operatorname{OBJ}(r)=T_{r}^{\mathrm{COST}}+\rho^{\mathrm{Q}} \cdot \max \left\{T_{r}^{\mathrm{Q}}-Q^{r}, 0\right\}+\rho^{\mathrm{TW}} \cdot T_{r}^{\mathrm{TW}}
$$

where $\rho^{\mathrm{Q}}$ is the weight of the load capacity violations and $\rho^{\mathrm{TW}}$ the time window violations. The objective value $\operatorname{OBJ}(s)$ of a solution $s$ is calculated as $\mathrm{OBJ}(s)=\Sigma_{r \in R(s)} \mathrm{OBJ}(r)$.

To compute the fitness of a solution for an individual in the population, we use the biased fitness function of the original HGA in Vidal et al. (2013), combining the objective value of an individual with its contribution to the population diversity so as to promote a good exploration of the search space. The diversity contribution 
of an individual $p$ is defined as the average distance to its $\mu^{\text {CLOSE }}$ most similar neighbors in the corresponding population $\mathcal{P}$, based on the broken-pairs distance (Prins, 2009). The biased fitness function $f_{\mathcal{P}}(p)$ is shown in Equation (5.3). It is the weighted sum of the objective cost rank $f_{\mathcal{P}}^{\text {oBJ }}(p)$ and its rank $f_{\mathcal{P}}^{\text {DIV }}(p)$ relative to its diversity contribution. The balance between these two terms is controlled by parameter $\mu^{\mathrm{ELITE}}$.

$$
f_{\mathcal{P}}(p)=f_{\mathcal{P}}^{\mathrm{OBJ}}(p)+\left(1-\frac{\mu^{\mathrm{ELITE}}}{|\mathcal{P}|}\right) \cdot f_{\mathcal{P}}^{\mathrm{DIV}}(p)
$$

\subsection{Generation of new individuals}

New individuals are created by recombination of two parent individuals, or by mutation, performed as a single destroy-and-recreate step on a single parent. Each parent is selected using a binary tournament scheme, where two random individuals from the pool of feasible and infeasible solutions are compared, and the better one in terms of biased fitness is chosen.

Recombination. To facilitate the recombination operation, the parent solutions are treated as giant tours, obtained by concatenating all the routes and omitting the visits to the depot. A giant tour is therefore simply an ordered permutation of all the customers. The giant tours of the parents are combined using the OX crossover (Prins, 2004; Vidal et al., 2012), to produce the giant tour of an offspring. Finally, a split algorithm is applied on this giant tour to optimally reinsert trip delimiters and define a complete solution. The split algorithm consists in the solution of shortest path problem in an auxiliary acyclic graph, in a similar manner as Prins (2004). Arcs for penalized infeasible routes $r$ are included in the auxiliary graph if $T_{r}^{\mathrm{Q}} \leq 2 Q^{r} \wedge T_{r}^{\mathrm{TW}} \leq 200$. The arc costs are obtained from the route evaluation operators presented in Section 4 , selecting the best vehicle type for each route.

Mutation. Our mutation operator consists in one step of a large neighborhood search, in which a subset of vertices is removed from the current solution and reinserted using a greedy heuristic. Similar techniques have been shown to be remarkably efficient for a variety of VRP variants (see, e.g., Ropke \& Pisinger, 2006; Christiaens \& Vanden Berghe, 2016; Hiermann et al., 2016). We rely on four classical removal operators:

- Random Removal: Select random vertices for removal;

- RANDom Routes: Select random routes for removal;

- Similar Removal: Iteratively, select a random vertex for removal along with one of its five closest customers (using the same measure as Section 5.3).

- Target Removal: As in Hiermann et al. (2016), select a vertex based on its contribution to the objective and remove the closest routes.

Each operator is repeated until at least $q$ nodes are removed, where $q$ is a number selected in $\left[\zeta^{\text {MIN }}, \zeta^{\text {Max }}\right]$ with uniform probability. Moreover, we opted to use a simple random selection of operators instead of an adaptive selection mechanism as in Ropke \& Pisinger (2006), as this simple scheme performed equally well in our preliminary experiments. Two operators are used for solution reconstruction with uniform probability: either a greedy insertion, or a 2-regret insertion. The greedy insertion finds the best route and position for each vertex, and performs the best insertion based on the objective function. The regret insertion uses a regret value to decide which vertex to insert, based on the difference between the best and the second best insertion positions. The evaluation of removals and insertions in the LNS uses an explicit route representation, in which recharging stations visits are explicitly listed. When evaluating the insertion cost of a vertex $i$, the method also attempts to jointly insert a recharging station with minimal detour, immediately before and/or after $i$, and keeps the best cost as in Hiermann et al. (2016). Our preliminary analyzes indicated that this explicit route representation in the LNS requires less computational effort than an implicit insertion during route evaluations, without any significant impact on solution quality.

\subsection{Improvement by local search}

The solution generated at each iteration using recombination, mutation, or set partitioning is improved using an LS procedure extended with a repair mechanism as proposed in Vidal et al. (2012). The LS scans the set of moves in random order. Any improving move is immediately applied, and the process is repeated 
until no such move can be found. If the improved solution is feasible (see Section 5.1), no additional action is performed. Otherwise, it is considered for a heuristic repair step with a probability of $p^{\text {REPAIR }}$, where the penalty weights are multiplied by 10 temporarily, and the LS is restarted. If it is still infeasible, another run with the weights multiplied by 100 is performed. No further attempts are made, and the local procedure terminates.

Neighborhood search. We use similar neighborhoods as the wide majority of state-of-the-art VRP metaheuristics (see, e.g., Vidal et al., 2012, 2013): 2-OPT, 2-OPT*, intra- and inter-route SwAP and RELOCATE, as well as VeHicle-Swap. The 2-OPT operator inverts a subsequence of visits within a single route. The 2-OPT* operator involves two routes: it splits each route into two parts and reconnects them differently. The SWAP and RELOCATE operators work by relocating or exchanging customer visits. We also apply ReLOCATE on pairs of consecutive visits, with a possible pair reversal. Finally, VEHICLE-SwAP move allows to change the vehicle type and class associated to any given route.

Neighborhood restrictions. We use static neighborhood restrictions to speed up the solution method. We calculate a set of so-called promising edges for each customer vertex (Vidal et al., 2013). Only the moves generating at least one promising edge are tested. Equation (5.4) defines a customer correlation measure, used to determine this set.

$$
\begin{array}{r}
\gamma(i, j)=d_{i j}+\gamma^{\mathrm{wT}} \cdot \max \left(e_{j}-s_{i}-t_{i j}-l_{i}, 0\right) \\
+\gamma^{\mathrm{TW}} \cdot \max \left(e_{i}+s_{i}+t_{i j}-l_{j}, 0\right)
\end{array}
$$

As seen in Equation (5.4), the set of promising edges from a customer $v_{i}$ is based on direct distance, unavoidable waiting time $\left(\gamma^{\mathrm{WT}}\right)$ and time-window violation $\left(\gamma^{\mathrm{TW}}\right)$. The final set of promising $\operatorname{arcs} \Gamma(i)$ consists of the $|\Gamma|$ closest customers with respect to the correlation measure $\gamma(i, j)$. The same setting as Vidal et al. (2013) is used: $|\Gamma|=40, \gamma^{\mathrm{WT}}=0.2$, and $\gamma^{\mathrm{TW}}=1.0$.

Lower bounds and memories. To avoid systematic calls to the computationally expensive DP procedure for BEV or PHEV route evaluations, we use lower bounds on move evaluations, as proposed in Vidal (2017), to filter a large proportion of candidate moves. To compute a fast lower bound, we first treat any BEV (PHEV) as a simple ICEV with a fuel cost identical to the electricity cost. Therefore, any move is first evaluated as an ICEV in $\mathcal{O}(1)$ operations, (effectively ignoring recharging stations) and compared to the current BEV (PHEV) route cost. If the move leads to a better objective, then the original BEV (PHEV) calculations are performed to determine the exact value of the move. Otherwise, the move cannot lead to a better solution and is discarded. Finally, a cache memory keeps track of move evaluations as long as the routes involved in the move are unchanged, so as to save unnecessary evaluations and CPU time.

\subsection{Population management}

We maintain two populations during the search, one with only feasible solutions and the other with only infeasible ones. Each population contains up to $\mu^{\mathrm{MIN}}+\mu^{\mathrm{GEN}}$ individuals. Whenever the population exceeds this number, a survivor-selection process is initialized to reduce the population to $\mu^{\mathrm{MIN}}$. This procedure sorts the solutions based on the biased fitness described in Section 5.1 and removes the last $\mu^{\mathrm{GEN}}$ individuals.

The two populations are initialized by generating $\mu^{\text {INIT }}$ solutions at the beginning of the search, applying local search and repair with a probability of $p$, and inserting them into the appropriate population. To diversify the search, a diversification phase is triggered after each $i t^{\text {DIV }}$ iterations without improvement of the best solutions. It removes all but the best $\mu^{\text {ELITE }}$ solutions and creates $\mu^{\text {div }}$ new random solutions, adding them to the corresponding population.

A solution is called naturally feasible if it is feasible after the LS procedure of Section 5.3. During the search, the penalties introduced in Section 5.1 are adapted to give a targeted proportion of naturally feasible solutions $\xi^{\mathrm{REF}}$. Let $\xi^{\mathrm{Q}}$ and $\xi^{\mathrm{TW}}$ be the proportion of naturally feasible solutions with regards to the load 
and time-window constraints respectively. The following adjustment is performed every 20 iterations, where $c \in\{\mathrm{Q}, \mathrm{TW}\}:$

$$
\rho^{\mathrm{C}}= \begin{cases}\rho^{\mathrm{C}} \cdot 1.20 & \text { if } \xi^{c} \leq \xi^{\mathrm{REF}}-0.05 \\ \rho^{\mathrm{C}} \cdot 0.85 & \text { if } \xi^{c} \geq \xi^{\mathrm{REF}}+0.05\end{cases}
$$

\subsection{Set partitioning}

The algorithm encounters many different routes during its search. To exploit this history, we embed a procedure to store and combine a subset of these routes. Such methods have been successfully applied in the past, leading to promising results for related problems (Subramanian et al., 2013).

At the end of every iteration of the HGA, the routes of the current local minimum are stored in an archive, together with the objective value and the overall solution quality. To avoid duplicates, a hash value is calculated based on the itinerary of the route, and the entry is replaced if a better route is encountered, i.e., using another vehicle class or type. To restrict the memory usage and computational effort, the size of this archive is bounded by $\lambda$. After a solution is added, if the bound is exceeded, around half of the stored routes are removed from the archive. This purge is biased based on the quality of the corresponding route. More details on the hashing calculation and the purging can be found in Goel \& Vidal (2014).

Every $i t^{\mathrm{SP}}$ iterations, a set partitioning problem is formulated with the routes available in the archive. The solution of this problem is a subset of routes with no overlapping customer assignments. We use partitioning instead of covering to avoid the need to repair overlapping assignments. The problem is solved using a commercial MIP solver. To improve the quality of the procedure, additional routes are generated during a preprocessing step with a single customer vertex; these are added to the formulation.

\section{Experiments}

Extensive experiments have been conducted in order to evaluate the performance of the HGA approach, and to gain managerial insights for the management of an hybrid fleet with different propulsion modes. All our experiments were run on a single thread of an Intel Xeon $26433.3 \mathrm{GHz}$ core, using a maximum RAM of 4 GB. We use CPLEX 12.6 for the solution of the set partitioning problems. The HGA was implemented in Java 8 and run using the Java Runtime Environment 1.8, Update 20 (JRE 8u20). We performed ten test runs for each instance, and report average and best results.

\section{1. $H^{2} E-F T W$ benchmark set}

We first created a $\mathrm{H}^{2} \mathrm{E}-\mathrm{FTW}$ benchmark set as a basis for the analysis of the metaheuristic and its components, as well as to allow for future comparisons. These instances are based on the 56 homogeneous electric fleet instances of Schneider et al. (2014), each with a single depot, 100 customers and 21 recharging stations. They are divided into six categories, based on their spatial (c, r, rc) and temporal (type-1, type-2) configurations. Instances of category (c) contain several clusters of customers. In (r) instances, customers are uniformly distributed, and (rc) instances consist of some clusters and some uniformly distributed customers. Type-1 instances have smaller time windows, whereas type-2 instances have larger ones as well as bigger vehicle capacities.

Each instance has been extended by adding a set of vehicle types and classes. The parameters of the vehicles are derived from a German study of the electric vehicle market (Plötz et al., 2013), in which the authors aggregated the different vehicle types into four categories: small, medium, large, and others. For these categories, average values are given for the parameters required in our work, e.g., consumption rate, battery size, and acquisition cost. We used the values of the first three categories (small, medium and large) for each of the three vehicle classes (ICEV, PHEV, BEV), leading to 9 vehicle types per instance.

Since the original E-VRPTW instances are based on artificial VRPTW instances, some adjustments had to be made. First, we used the properties provided in the E-VRPTW instance files and assumed that the original vehicle is in the "large" category. The remaining vehicles types were derived by fixing this baseline and using the relative differences from Plötz et al. (2013). Finally all distances were scaled in such a way that one unit of distance represents one kilometer. 


\subsection{Parameter calibration}

Subsequently, we performed a calibration of the method's parameters. To avoid over-tuning, we used a randomly selected subset of instances: c101, r104, rc102, rc108, c203, r202, r205, and rc202. We identified a subset of parameters with a larger effect on the performance of the algorithm. These are: the population size $\mu$, the number of iterations $i t^{\mathrm{MAX}}$, the crossover probability $p^{\mathrm{CRoss}}$ (which is the same as the crossoverto-mutation ratio), the number of nonimproving iterations before diversifying $i t^{\mathrm{DIV}}$, and the diversification ratio $\mu^{\text {DIV }} / \mu$.

We first conducted preliminary experiments to converge towards suitable values for these parameters, leading us to the configuration marked with an asterisk $\left(^{*}\right)$ in Table 6.1. Then, we varied each parameter, one factor at time (OFAT approach), while fixing the others. This led us to several alternative configurations which are also reported in the table. These results allow to visualize the individual impact of each parameter, and highlights some clear trade-offs between solution quality and CPU time. The final parameters were selected to balance these two performance indicators, as shown in Table 6.2.

\begin{tabular}{l|rrrr}
\hline$\left(\mu^{\mathrm{MIN}}, \mu^{\mathrm{GEN}}\right)$ & $(3,1)$ & $(5,3)$ & $(10,5)^{*}$ & $(17,8)$ \\
$\mathrm{obj}$ & 1945.99 & 1944.75 & $\mathbf{1 9 4 3 . 5 9}$ & 1945.14 \\
$\mathrm{t}[\mathrm{s}]$ & $\mathbf{6 9 1 . 7 5}$ & 751.04 & 854.58 & 1013.92 \\
\hline$i t^{\mathrm{MAX}}$ & 500 & 1000 & $1500^{*}$ & 2000 \\
$\mathrm{obj}$ & 1966.65 & 1953.18 & 1943.59 & $\mathbf{1 9 4 2 . 4 8}$ \\
$\mathrm{t}[\mathrm{s}]$ & $\mathbf{2 6 1 . 4 5}$ & 557.27 & 854.58 & 1170.02 \\
\hline$p^{\mathrm{CROSs}}$ & 0.2 & $0.4^{*}$ & 0.6 & 0.8 \\
$\mathrm{obj}$ & 1951.56 & 1943.59 & 1939.44 & $\mathbf{1 9 3 4 . 3 5}$ \\
$\mathrm{t}[\mathrm{s}]$ & $\mathbf{7 8 5 . 2 8}$ & 854.58 & 931.15 & 972.97 \\
\hline$i t^{\mathrm{DIV}}$ & 50 & $100^{*}$ & 200 & 500 \\
$\mathrm{obj}$ & 1944.38 & 1943.59 & $\mathbf{1 9 4 1 . 3 6}$ & 1948.55 \\
$\mathrm{t}[\mathrm{s}]$ & 1075.62 & 854.58 & 733.69 & $\mathbf{7 0 5 . 2 9}$ \\
\hline$\mu^{\mathrm{DIV}} / \mu$ & 0.5 & $1 *$ & 1.5 & 2 \\
$\mathrm{obj}$ & 1944.73 & $\mathbf{1 9 4 3 . 5 9}$ & 1946.31 & 1944.23 \\
$\mathrm{t}[\mathrm{s}]$ & $\mathbf{7 7 2 . 7 4}$ & 854.58 & 950.34 & 1034.05 \\
\hline
\end{tabular}

Table 6.1: Parameter calibration results from settings derived from preliminary experiments. Values marked with * were used as the base setting for this setup.

\begin{tabular}{lr|lr}
\hline$i t^{\mathrm{MAX}}$ & 1500 & $\mu^{\mathrm{MIN}}$ & 5 \\
$i t^{\mathrm{SP}}$ & 25 & $\mu^{\mathrm{GEN}}$ & 3 \\
$t^{\mathrm{SP}}$ & $10 \mathrm{~s}$ & $\mu^{\mathrm{ELITE}}$ & 3 \\
$p^{\mathrm{CROSS}}$ & 0.4 & $i t^{\text {DIV }}$ & 200 \\
$p^{\mathrm{REPAIR}}$ & 0.5 & $\mu^{\text {DIV }} / \mu$ & 0.5 \\
$\zeta^{\mathrm{MAX}}$ & 10 & & \\
$\zeta^{\text {MIN }}$ & 30 & & \\
\hline
\end{tabular}

Table 6.2: Parameter settings after calibration.

Finally, for the experiments with different cost values for fuel and electricity usage, we will consider the following scenarios: the electric energy cost can be $\{0.1,0.3,0.5,0.7\}$ monetary units per unit $(\mathrm{kW})$ consumed, and fuel cost can be $\{1.0,1.5,2.0,2.5,3.0,4.0\}$ monetary units per unit (liter).

\subsection{Sensitivity Analysis - Components of the Method}

Table 6.3 compares different compositions of the method components described in Section 5. The first three rows show which components are active in the corresponding run. The fourth row reports the average results over all ten runs, and the fifth row gives the average CPU time in seconds. The adjacent percentage gives the deviation from the base setting (first column). Deactivating any component leads to a deterioration of up to $3 \%$ of the average objective value found by the complete method. The time differences are larger: deactivating the crossover results in a decrease of $26 \%$. On the other hand, deactivating the LNS gives similar results in terms of objective value but increases the CPU time by $15 \%$. We therefore use the complete method, with crossover, LNS and set partitioning, for the remainder of these experiments. 


\begin{tabular}{|c|c|c|c|c|c|c|c|c|c|c|c|}
\hline $\begin{array}{l}\text { Cross } \\
\text { LNS } \\
\text { SP }\end{array}$ & $\begin{array}{l}X \\
X \\
X\end{array}$ & \multicolumn{2}{|c|}{$\mathrm{X}$} & \multicolumn{2}{|c|}{$X$} & \multicolumn{2}{|c|}{$\begin{array}{l}X \\
X\end{array}$} & \multicolumn{2}{|c|}{$\begin{array}{l}\mathrm{X} \\
\mathrm{X}\end{array}$} & \multicolumn{2}{|c|}{$\begin{array}{l}\mathrm{X} \\
\mathrm{X}\end{array}$} \\
\hline $\begin{array}{l}o b j_{a v g} \\
t[s]\end{array}$ & $\begin{array}{l}1701.03 \\
1097.08\end{array}$ & $\begin{array}{l}1743.87 \\
1193.86\end{array}$ & $\begin{array}{l}2.52 \% \\
8.82 \%\end{array}$ & $\begin{array}{r}1752.46 \\
811.89\end{array}$ & $\begin{array}{r}3.02 \% \\
-26.00 \%\end{array}$ & $\begin{array}{r}1728.21 \\
884.48\end{array}$ & $\begin{array}{r}1.60 \% \\
-23.03 \%\end{array}$ & $\begin{array}{l}1701.26 \\
1257.89\end{array}$ & $\begin{array}{r}0.01 \% \\
14.66 \%\end{array}$ & $\begin{array}{l}1725.77 \\
1050.97\end{array}$ & $\begin{array}{r}1.45 \% \\
-4.20 \%\end{array}$ \\
\hline
\end{tabular}

Table 6.3: Analysis of the components involved, showing the average objective value and CPU time. The percentages next to the values show the deviation from the base setting (left column).

\subsection{Sensitivity Analysis - Fleet composition}

We have formulated a fleet mix problem with different vehicle classes, ICEV, PHEV, and BEV. To assess the benefits of fleet-mix optimization and the impact of different vehicle classes, we performed experiments restricting the fleet to a single class. Table 6.4 and Figure 6.1 show this comparison. For each cost value of the respective metric $\left(c_{\mathrm{E}}\right.$ - electricity cost, $c_{\mathrm{F}}$ - fuel cost $) 10$ runs of each single class case are compared to runs from the mixed case.

\begin{tabular}{|c|c|c|c|c|c|c|c|c|c|c|c|c|c|}
\hline & & \multicolumn{3}{|c|}{ All } & \multicolumn{3}{|c|}{ ICEV } & \multicolumn{3}{|c|}{$\mathrm{BEV}$} & \multicolumn{3}{|c|}{ PHEV } \\
\hline & & Obj & Gap & \#Veh & Obj & Gap & \#Veh & Obj & Gap & \#Veh & Obj & Gap & \#Veh \\
\hline \multirow[t]{4}{*}{$c_{\mathrm{E}}$} & 0.10 & 1543.69 & $0.00 \%$ & 8.17 & 1932.88 & $25.21 \%$ & 7.95 & 1637.46 & $6.07 \%$ & 8.06 & 1660.90 & $7.59 \%$ & 9.27 \\
\hline & 0.30 & 1683.28 & $0.00 \%$ & 7.81 & 1933.67 & $14.88 \%$ & 7.96 & 1806.49 & $7.32 \%$ & 7.99 & 1808.51 & $7.44 \%$ & 8.90 \\
\hline & 0.50 & 1770.44 & $0.00 \%$ & 7.56 & 1937.95 & $9.46 \%$ & 7.97 & 1986.22 & $12.19 \%$ & 7.91 & 1930.59 & $9.05 \%$ & 8.69 \\
\hline & 0.70 & 1816.56 & $0.00 \%$ & 7.43 & 1931.98 & $6.35 \%$ & 7.94 & 2199.41 & $21.08 \%$ & 7.89 & 2032.41 & $11.88 \%$ & 8.65 \\
\hline \multirow[t]{6}{*}{$c_{\mathrm{F}}$} & 1.00 & 1445.51 & $0.00 \%$ & 7.29 & 1527.95 & $5.70 \%$ & 7.71 & 1909.96 & $32.13 \%$ & 7.79 & 1641.40 & $13.55 \%$ & 8.91 \\
\hline & 1.50 & 1569.98 & $0.00 \%$ & 7.41 & 1686.38 & $7.41 \%$ & 7.83 & 1897.81 & $20.88 \%$ & 7.82 & 1734.58 & $10.48 \%$ & 8.82 \\
\hline & 2.00 & 1679.62 & $0.00 \%$ & 7.56 & 1837.54 & $9.40 \%$ & 7.91 & 1884.77 & $12.21 \%$ & 7.88 & 1822.17 & $8.49 \%$ & 8.76 \\
\hline & 2.50 & 1768.62 & $0.00 \%$ & 7.77 & 1991.89 & $12.62 \%$ & 7.97 & 1912.76 & $8.15 \%$ & 7.97 & 1899.43 & $7.40 \%$ & 8.88 \\
\hline & 3.00 & 1838.10 & $0.00 \%$ & 8.00 & 2135.65 & $16.19 \%$ & 8.05 & 1910.79 & $3.95 \%$ & 8.08 & 1967.41 & $7.03 \%$ & 8.90 \\
\hline & 4.00 & 1919.12 & $0.00 \%$ & 8.42 & 2425.31 & $26.38 \%$ & 8.24 & 1928.28 & $0.48 \%$ & 8.25 & 2083.62 & $8.57 \%$ & 9.00 \\
\hline
\end{tabular}

Table 6.4: Comparison of results for individual vehicle classes and all classes. Each row of the electric cost setting $\left(c_{e}\right)$ reports the average value over all runs with all fuel cost settings, and vice versa.
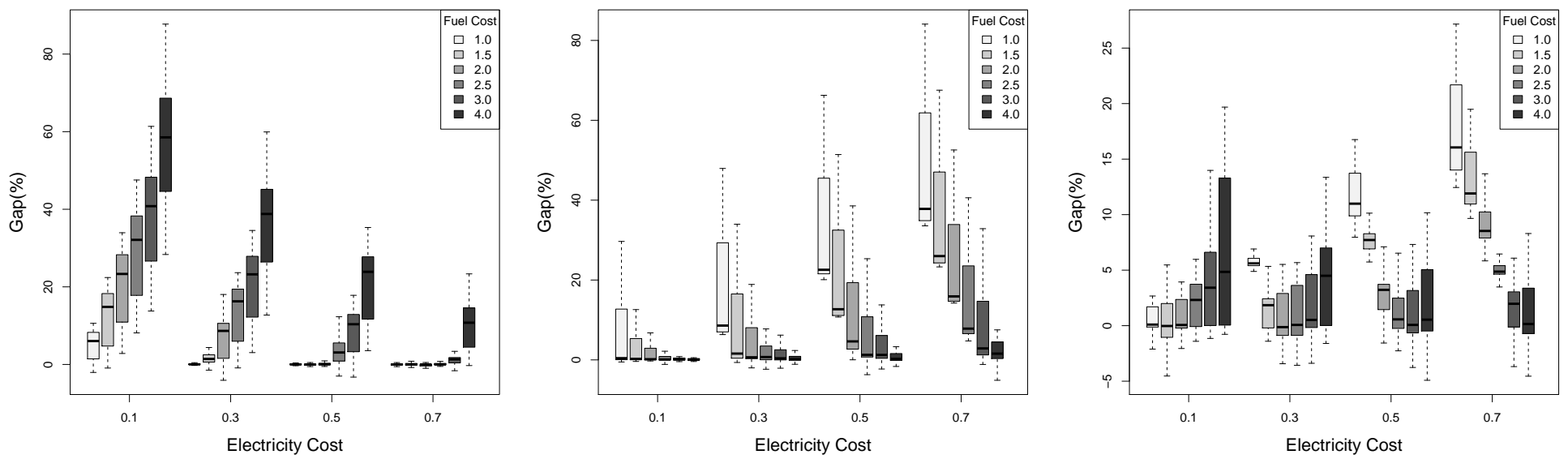

Figure 6.1: $\operatorname{Gap}(\%)$ of the solutions obtained with an homogeneous fleet of ICEV (left), BEV (middle), and PHEV (right), relative to the optimized solution with a mixed fleet.

The gap for the ICEV case is growing with increasing fuel cost with more than $60 \%$ on average for the extreme case $\left(c_{\mathrm{E}}=0.1, c_{\mathrm{F}}=1.0\right)$. BEV gaps grow with increasing electricity costs, up around $40 \%$ on average. In case of PHEV, the gaps remain rather small for most settings. However, in case of either low 
electricity or low fuel cost, the gap increases up to $25 \%$. This can be due to the higher fixed cost of PHEVs compared to the other classes.

In addition, Table 6.5 shows detailed results for a single cost setting $\left(c_{\mathrm{E}}=0.3, c_{\mathrm{F}}=2.0\right)$. We chose the one with the most evenly spread fleet mix on average. In these results we observe that BEVs are preferred for the clustered (c) type instances, where the distances between customers are usually smaller. This gives an advantage to the cheaper but distance-limited BEVs. The inverse tendency can be observed for the randomly distributed (r) instances, where ICEVs are used in majority. A fairly balanced use of all vehicle classes is observable in the (rc) type instances. Finally, PHEVs can be preferred over the other vehicle classes for randomly distributed (r) and random-clustered (rc) instances of type 2.

\begin{tabular}{|c|c|c|c|c|c|c|c|c|c|c|c|c|c|}
\hline Name & Best 10 & Avg 10 & $\mathrm{~T}(\min )$ & I & $\mathrm{P}$ & B & Name & Best 10 & Avg 10 & $\mathrm{~T}(\min )$ & I & $\mathrm{P}$ & B \\
\hline c101 & 2484.52 & 2486.10 & 6.47 & 0.4 & 0.8 & 10.8 & $\mathrm{c} 201$ & 832.80 & 833.81 & 11.89 & 0.0 & 0.0 & 4.0 \\
\hline c102 & 2324.03 & 2380.20 & 7.40 & 1.6 & 1.2 & 8.1 & c202 & 830.82 & 833.19 & 13.76 & 0.0 & 0.0 & 4.0 \\
\hline c103 & 2290.92 & 2317.66 & 8.35 & 1.1 & 2.5 & 6.8 & c203 & 827.49 & 828.60 & 16.22 & 0.0 & 0.0 & 4.0 \\
\hline c104 & 2209.32 & 2215.73 & 9.47 & 1.3 & 2.0 & 6.7 & c204 & 823.84 & 823.98 & 16.88 & 0.0 & 0.0 & 4.0 \\
\hline c105 & 2373.39 & 2410.22 & 6.78 & 1.4 & 3.1 & 6.3 & c205 & 824.43 & 824.43 & 12.97 & 0.0 & 0.0 & 4.0 \\
\hline c106 & 2372.68 & 2404.75 & 7.27 & 1.3 & 2.6 & 7.0 & c206 & 824.57 & 824.57 & 14.29 & 0.0 & 0.0 & 4.0 \\
\hline c107 & 2351.41 & 2396.50 & 6.93 & 1.4 & 2.2 & 7.3 & $\mathrm{c} 207$ & 824.18 & 824.18 & 14.30 & 0.0 & 0.0 & 4.0 \\
\hline c108 & 2321.09 & 2346.54 & 7.70 & 1.6 & 3.5 & 5.2 & c208 & 824.64 & 824.64 & 13.72 & 0.0 & 0.0 & 4.0 \\
\hline c109 & 2248.84 & 2277.38 & 7.72 & 0.7 & 2.8 & 6.7 & & & & & & & \\
\hline r101 & 3561.46 & 3602.19 & 7.57 & 12.4 & 1.2 & 2.7 & r201 & 640.68 & 642.29 & 18.00 & 0.0 & 3.0 & 0.0 \\
\hline r102 & 3240.81 & 3258.85 & 8.05 & 10.7 & 1.4 & 2.9 & r202 & 582.49 & 583.14 & 19.72 & 0.0 & 3.0 & 0.0 \\
\hline r103 & 2781.05 & 2818.80 & 8.63 & 9.6 & 1.0 & 1.7 & r203 & 592.12 & 593.93 & 23.98 & 0.0 & 3.0 & 0.0 \\
\hline r104 & 2437.22 & 2468.18 & 10.23 & 7.3 & 1.5 & 1.6 & r204 & 425.81 & 427.67 & 39.59 & 0.0 & 2.0 & 0.0 \\
\hline r105 & 2979.09 & 2991.25 & 7.77 & 10.6 & 1.0 & 1.4 & r205 & 597.00 & 598.67 & 25.57 & 0.0 & 3.0 & 0.0 \\
\hline r106 & 2806.32 & 2817.29 & 9.04 & 9.9 & 1.5 & 0.6 & r206 & 600.59 & 601.79 & 31.11 & 0.0 & 3.0 & 0.0 \\
\hline r107 & 2456.63 & 2528.41 & 9.14 & 7.8 & 0.9 & 1.9 & $\mathrm{r} 207$ & 425.55 & 427.86 & 38.82 & 0.0 & 2.0 & 0.0 \\
\hline r108 & 2329.04 & 2385.74 & 9.43 & 7.8 & 0.8 & 1.2 & r208 & 432.17 & 433.94 & 40.03 & 0.0 & 2.0 & 0.0 \\
\hline r109 & 2616.63 & 2651.03 & 7.78 & 8.6 & 1.0 & 1.6 & $\mathrm{r} 209$ & 592.21 & 593.73 & 29.99 & 0.0 & 3.0 & 0.0 \\
\hline r110 & 2389.62 & 2449.35 & 9.35 & 8.3 & 0.3 & 1.7 & r210 & 585.77 & 586.92 & 32.27 & 0.0 & 3.0 & 0.0 \\
\hline r111 & 2436.30 & 2479.86 & 9.37 & 8.2 & 1.1 & 0.9 & $\mathrm{r} 211$ & 425.90 & 443.39 & 44.17 & 0.0 & 2.0 & 0.1 \\
\hline $\mathrm{r} 112$ & 2366.37 & 2382.81 & 9.28 & 8.1 & 1.1 & 0.8 & & & & & & & \\
\hline rc101 & 3159.62 & 3212.67 & 7.53 & 6.1 & 2.1 & 6.5 & rc201 & 800.61 & 801.43 & 18.18 & 0.0 & 4.0 & 0.0 \\
\hline rc102 & 2974.32 & 3029.84 & 8.02 & 4.9 & 3.7 & 5.0 & rc202 & 618.81 & 620.69 & 23.07 & 0.0 & 2.9 & 0.1 \\
\hline rc103 & 2644.37 & 2712.23 & 8.48 & 4.7 & 3.0 & 4.2 & rc203 & 617.31 & 620.22 & 24.79 & 0.0 & 3.0 & 0.0 \\
\hline rc104 & 2423.23 & 2459.12 & 9.47 & 4.3 & 3.7 & 2.2 & rc204 & 634.61 & 638.10 & 26.13 & 0.0 & 2.9 & 0.1 \\
\hline rc105 & 2889.19 & 2907.22 & 7.65 & 5.2 & 3.2 & 4.6 & rc205 & 659.20 & 695.54 & 20.95 & 0.2 & 2.3 & 0.5 \\
\hline rc106 & 2761.25 & 2791.56 & 8.12 & 5.4 & 3.6 & 3.2 & rc206 & 622.83 & 625.01 & 24.25 & 0.0 & 3.0 & 0.0 \\
\hline rc107 & 2543.54 & 2571.74 & 8.71 & 4.5 & 3.6 & 3.0 & rc207 & 609.47 & 613.20 & 30.83 & 0.0 & 2.9 & 0.1 \\
\hline rc108 & 2446.58 & 2485.15 & 8.70 & 3.3 & 3.6 & 3.8 & rc208 & 623.79 & 626.71 & 34.28 & 0.0 & 2.5 & 0.5 \\
\hline
\end{tabular}

Table 6.5: Detailed results for each instance with fixed costs (electricity: 0.30, fuel: 2.00): best and average objective value, average CPU time in minutes, and average number of vehicles per class (I: ICEV, P: PHEV, and B: BEV).

\subsection{Sensitivity Analysis - Cost factors}

\begin{tabular}{l|cccccc}
\hline$c_{\mathrm{E}} \backslash c_{\mathrm{F}}$ & 1.00 & 1.50 & 2.00 & 2.50 & 3.00 & 4.00 \\
\hline 0.10 & $-3.00 \%$ & $1.28 \%$ & $3.59 \%$ & $4.93 \%$ & $5.83 \%$ & $6.69 \%$ \\
0.30 & $0.01 \%$ & $8.75 \%$ & $14.58 \%$ & $18.09 \%$ & $19.82 \%$ & $21.72 \%$ \\
0.50 & $-0.05 \%$ & $9.52 \%$ & $19.31 \%$ & $26.63 \%$ & $31.24 \%$ & $36.17 \%$ \\
0.70 & $0.00 \%$ & $9.55 \%$ & $19.43 \%$ & $28.81 \%$ & $37.64 \%$ & $47.65 \%$ \\
\hline
\end{tabular}

Table 6.6: Gap in the objective value for different cost values compared to the cost combination (electricity: 0.70 , fuel: 1.00).

We now investigate the impact of fuel and electricity cost on optimized fleet compositions and operational costs. Table 6.6 shows the average gap in the objective value compared to high electricity cost, low fuel cost scenario $\left(c_{\mathrm{E}}=0.7, c_{\mathrm{F}}=1.0\right)$. Figure 6.2 presents the corresponding average vehicle class usage over all runs of all instances.

The gap is nearly zero in all other electricity cost settings but the smallest with 0.1 , where the gap is negative with $-3 \%$. By using a higher fuel cost value of 4.0 , the gap increases to $47.65 \%$. The increase is 


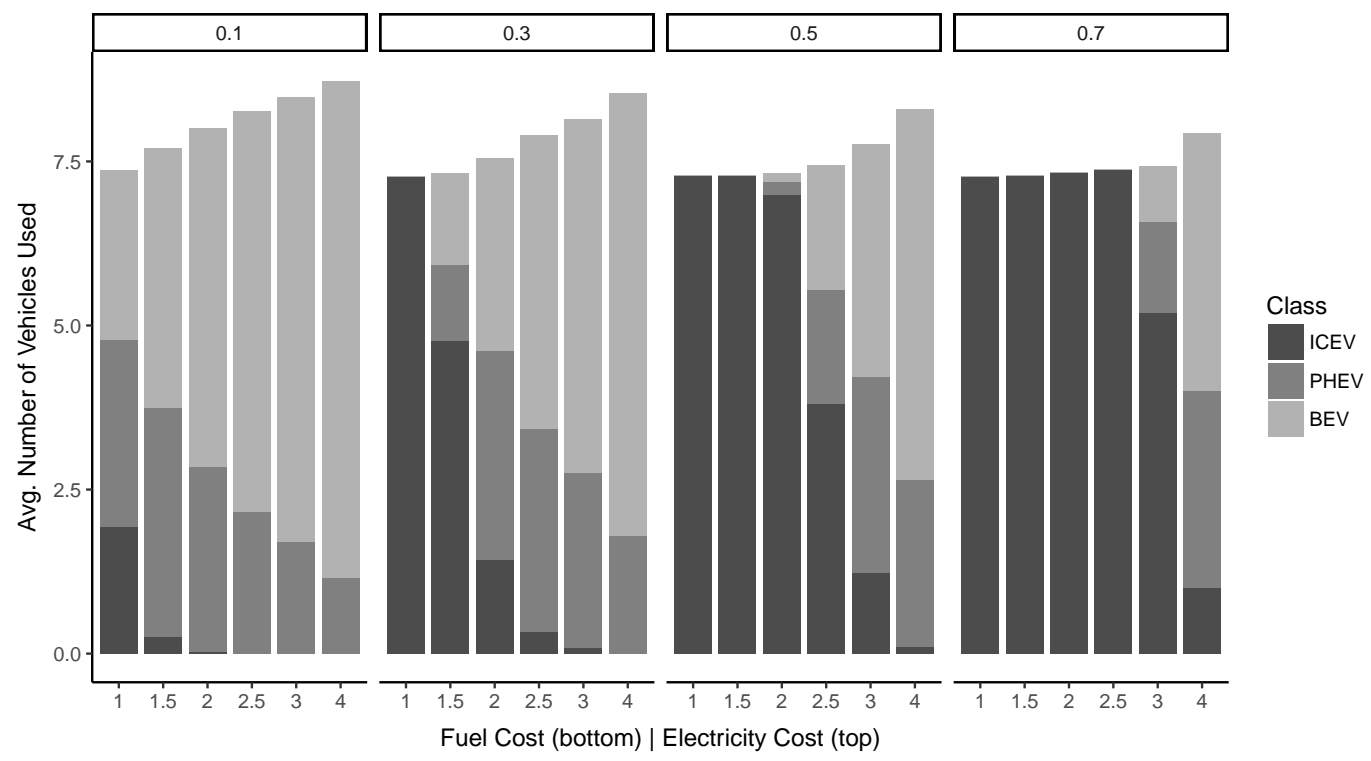

Figure 6.2: Change in the fleet composition for different cost values.

steeper in the the lower value settings, with almost $10 \%$ per step, e.g., in scenario $\left(c_{\mathrm{E}}=0.7, c_{\mathrm{F}}=1.5\right)$ and $\left(c_{\mathrm{E}}=0.7, c_{\mathrm{F}}=2.0\right)$. The reason for this can be observed in Figure 6.2. In the low fuel cost scenarios with higher electricity cost $\left(c_{\mathrm{E}}=0.5, c_{\mathrm{E}}=0.7\right)$, the number of vehicles is not increasing and almost all vehicles in the mix are ICEVs. The gap increase is caused only by the increase in the fuel cost. When the increase is less steep, e.g., between scenario $\left(c_{\mathrm{E}}=0.5, c_{\mathrm{F}}=2.0\right)$ and $\left(c_{\mathrm{E}}=0.5, c_{\mathrm{F}}=2.5\right)$ with around $7 \%$, we can observe a shift in the fleet mix towards electric vehicles.

We can also observe the inverse with the electricity cost where an increase leads to a full switch to an ICEV-only fleet. This happens instantly between scenario $\left(c_{\mathrm{E}}=0.1, c_{\mathrm{F}}=1.0\right)$ and $\left(c_{\mathrm{E}}=0.3, c_{\mathrm{F}}=1.0\right)$. In these scenarios the higher utility costs of BEVs become a key factor compared to the very cheap fuel.

Note that PHEVs are rarely the largest component of a fleet on average. This may be due to their generally higher utility cost and the higher consumption rates compared to similar ICEVs and BEVs. However, due to their increased flexibility, they still play a role in many optimized fleet configurations, representing $20 \%$ of the overall number of vehicles used in the solutions.

\subsection{Performance Analysis - E-VRPTW(PR)}

The E-VRPTW is the seminal problem in the field of electric vehicle routing with recharging stations. It defines a fixed recharging policy, where the BEV is always recharged to full capacity at a recharging station. This assumption was relaxed by Keskin \& Çatay (2016), resulting in the E-VRPTW with partial recharging (E-VRPTWPR). Both formulations aim first to optimize the number of vehicles used and then to decrease the total distance of the routes. To achieve this, we assigned a high fixed cost $\left(f^{k}=2000\right)$ for the use of a vehicle. Moreover, minor modifications were applied to the route-evaluation layers to comply with the fixed recharging scheme. To calculate the objective value, we use the extension functions described in Hiermann et al. (2016). The dominance criterion is also adapted accordingly.

Table 6.7 and 6.8 show the results for the E-VRPTW and E-VRPTWPR benchmark instances. The best known solutions (BKS) prior to this work are shown in the right-side column. The bottom row (Gap) presents the average deviation from the best known solutions (i.e., Gap $\left.=\left(\mathrm{obj}^{-} \mathrm{obj}^{\mathrm{BKS}}\right) / \mathrm{obj}^{\mathrm{BKS}}\right)$. Our results are similar to those in the literature, on average within around $0.2 \%$ of the best known solutions. We found 11 new best solutions, the majority in the type-1 category, which has tighter time windows.

The average CPU time of our solver is 11.59 min for the E-VRPTW. This performance is competitive; in the literature, time values of 15.34 min (Schneider et al., 2014), 15.92 min (Hiermann et al., 2014), and 


\begin{tabular}{|c|c|c|c|c|c|c|c|c|}
\hline \multirow[t]{2}{*}{ Name } & \multirow{2}{*}{$\begin{array}{c}\text { SSG } \\
\text { Best } 10\end{array}$} & \multirow{2}{*}{$\begin{array}{c}\text { HPH } \\
\text { Best } 10\end{array}$} & \multirow{2}{*}{$\begin{array}{c}\text { GS } \\
\text { Best } 10\end{array}$} & \multirow{2}{*}{$\begin{array}{c}\text { KÇ } \\
\text { Best } 10\end{array}$} & \multicolumn{3}{|c|}{ HGA } & \multirow[t]{2}{*}{ BKS } \\
\hline & & & & & Avg 10 & Best 10 & $\mathrm{~T}(\min )$ & \\
\hline c101 & $12 / 1053.83$ & $12 / 1053.83$ & $12 / 1053.83$ & $12 / 1053.83$ & $12.0 / 1053.83$ & $12 / 1053.83$ & 3.81 & $12 / 1053.83$ \\
\hline $\mathrm{c} 102$ & $11 / 1057.21$ & $11 / 1057.21$ & $11 / 1057.21$ & $11 / 1056.12$ & $11.0 / 1057.45$ & $11 / 1055.12$ & 5.37 & $11 / 1051.38$ \\
\hline c103 & $10 / 1041.55$ & $10 / 1044.15$ & $10 / 1038.84$ & $11 / 1001.81$ & $10.6 / 1020.22$ & $10 / 1034.86$ & 9.93 & $10 / 1034.86$ \\
\hline c104 & $10 / 980.78$ & $10 / 984.60$ & $10 / 972.07$ & $10 / 951.57$ & $10.0 / 958.91$ & $10 / 953.63$ & 10.07 & $10 / 951.57$ \\
\hline c105 & $11 / 1075.37$ & $11 / 1075.37$ & $11 / 1075.37$ & $11 / 1075.37$ & $11.0 / 1076.71$ & $11 / 1075.37$ & 4.65 & $11 / 1075.37$ \\
\hline c106 & $11 / 1057.86$ & $11 / 1057.65$ & $11 / 1057.65$ & $11 / 1057.65$ & $11.0 / 1058.17$ & $11 / 1057.65$ & 5.25 & $11 / 1057.65$ \\
\hline c107 & $11 / 1031.56$ & $11 / 1031.56$ & $11 / 1031.56$ & $11 / 1031.56$ & $11.0 / 1033.44$ & $11 / 1031.56$ & 7.00 & $11 / 1031.56$ \\
\hline c109 & $10 / 1051.88$ & $10 / 1051.46$ & $10 / 1033.63$ & $10 / 1069.16$ & $10.4 / 1044.71$ & $10 / 1060.78$ & 11.26 & $10 / 1033.67$ \\
\hline $\mathrm{c} 201$ & $4 / 645.16$ & $4 / 645.16$ & $4 / 645.16$ & $4 / 645.16$ & $4.0 / 645.16$ & $4 / 645.16$ & 5.38 & 4/645.16 \\
\hline $\mathrm{c} 202$ & $4 / 645.16$ & $4 / 646.51$ & $4 / 645.16$ & $4 / 645.16$ & $4.0 / 645.16$ & $4 / 645.16$ & 7.59 & $4 / 645.16$ \\
\hline $\mathrm{c} 203$ & $4 / 644.98$ & $4 / 644.98$ & $4 / 644.98$ & $4 / 644.98$ & $4.0 / 645.00$ & $4 / 644.98$ & 7.93 & $4 / 644.98$ \\
\hline c204 & $4 / 636.43$ & $4 / 638.34$ & $4 / 636.43$ & $4 / 636.43$ & $4.0 / 636.92$ & $4 / 636.43$ & 8.57 & $4 / 636.43$ \\
\hline $\mathrm{c} 205$ & $4 / 641.13$ & $4 / 641.13$ & $4 / 641.13$ & $4 / 641.13$ & $4.0 / 641.13$ & $4 / 641.13$ & 6.46 & $4 / 641.13$ \\
\hline c206 & $4 / 638.17$ & $4 / 638.17$ & $4 / 638.17$ & $4 / 638.17$ & $4.0 / 638.17$ & $4 / 638.17$ & 7.32 & $4 / 638.17$ \\
\hline c207 & $4 / 638.17$ & $4 / 638.17$ & $4 / 638.17$ & $4 / 638.17$ & $4.0 / 638.17$ & $4 / 638.17$ & 7.56 & $4 / 638.17$ \\
\hline c208 & $4 / 638.17$ & $4 / 638.17$ & $4 / 638.17$ & $4 / 638.17$ & $4.0 / 638.17$ & $4 / 638.17$ & 7.54 & $4 / 638.17$ \\
\hline r102 & $16 / 1535.87$ & $16 / 1488.97$ & $16 / 1492.84$ & $16 / 1519.80$ & $16.0 / 1486.99$ & $16 / 1484.57$ & 7.95 & $16 / 1487.41$ \\
\hline r103 & $13 / 1299.59$ & $13 / 1285.96$ & $13 / 1281.97$ & $13 / 1312.50$ & $\overline{13.2 / 1284.47}$ & $13 / 1268.88$ & 11.89 & $13 / 1271.35$ \\
\hline r104 & $11 / 1088.43$ & $11 / 1097.79$ & $11 / 1090.72$ & $12 / 1071.89$ & $11.7 / 1090.68$ & $\overline{11 / 1103.50}$ & 14.74 & $11 / 1088.43$ \\
\hline r105 & $14 / 1473.52$ & $15 / 1433.92$ & $14 / 1453.80$ & $15 / 1383.29$ & $15.0 / 1394.63$ & $15 / 1382.70$ & 13.56 & $14 / 1442.35$ \\
\hline r106 & $13 / 1344.66$ & $13 / 1363.22$ & $13 / 1359.85$ & $14 / 1276.15$ & $14.0 / 1275.88$ & $14 / 1275.86$ & 14.95 & $13 / 1324.10$ \\
\hline r107 & $12 / 1154.52$ & $12 / 1165.37$ & $12 / 1151.52$ & $12 / 1148.43$ & $12.0 / 1163.54$ & $12 / 1148.38$ & 13.78 & $12 / 1148.43$ \\
\hline r108 & $11 / 1065.90$ & $11 / 1067.47$ & $11 / 1062.85$ & $11 / 1051.59$ & $11.0 / 1064.58$ & $11 / 1049.12$ & 15.23 & $11 / 1050.04$ \\
\hline r109 & $12 / 1294.05$ & $13 / 1245.91$ & $12 / 1261.31$ & $13 / 1214.72$ & $13.0 / 1228.37$ & $\overline{13 / 1214.63}$ & 14.43 & $12 / 1261.31$ \\
\hline r110 & $11 / 1143.53$ & $11 / 1155.58$ & $11 / 1141.39$ & $12 / 1097.89$ & $12.0 / 1103.46$ & $12 / 1097.66$ & 15.22 & $11 / 1119.50$ \\
\hline r111 & $12 / 1124.11$ & $12 / 1120.46$ & $12 / 1124.00$ & $12 / 1109.14$ & $12.0 / 1111.12$ & $12 / 1099.53$ & 14.72 & $12 / 1106.19$ \\
\hline r112 & $11 / 1026.52$ & $11 / 1043.77$ & $11 / 1026.73$ & $11 / 1038.74$ & $11.1 / 1023.78$ & $11 / 1016.63$ & 14.25 & $11 / 1016.63$ \\
\hline $\mathrm{r} 201$ & $3 / 1264.82$ & $3 / 1269.50$ & $3 / 1267.48$ & $3 / 1265.67$ & $3.0 / 1273.88$ & $3 / 1267.14$ & 9.81 & $3 / 1264.82$ \\
\hline r202 & $3 / 1052.32$ & $3 / 1053.90$ & $3 / 1052.85$ & 3/1052.32 & $3.0 / 1053.45$ & $3 / 1052.32$ & 10.96 & $3 / 1052.32$ \\
\hline r203 & 3/912.84 & $3 / 897.16$ & $3 / 899.05$ & $3 / 895.54$ & $3.0 / 910.15$ & $3 / 895.54$ & 12.35 & $3 / 895.54$ \\
\hline r204 & $2 / 790.56$ & $2 / 788.67$ & $2 / 782.91$ & $2 / 780.98$ & $2.0 / 795.91$ & $2 / 784.77$ & 19.19 & $2 / 779.49$ \\
\hline $\mathrm{r} 205$ & $3 / 988.67$ & $3 / 1002.02$ & $3 / 990.05$ & $3 / 987.36$ & $3.0 / 995.02$ & $3 / 987.36$ & 11.51 & $3 / 987.36$ \\
\hline r206 & $3 / 925.19$ & $3 / 922.70$ & $3 / 924.82$ & $3 / 922.70$ & $3.0 / 932.97$ & $3 / 925.34$ & 14.18 & $3 / 922.19$ \\
\hline r207 & $2 / 852.69$ & $2 / 859.82$ & $2 / 848.95$ & $2 / 847.14$ & $2.0 / 851.73$ & $2 / 847.59$ & 18.08 & $2 / 845.26$ \\
\hline $\mathrm{r} 210$ & $3 / 847.06$ & $3 / 863.49$ & $3 / 849.94$ & $3 / 843.65$ & $3.0 / 850.48$ & $3 / 846.62$ & 17.86 & $3 / 843.65$ \\
\hline $\mathrm{r} 211$ & $2 / 866.18$ & $2 / 873.64$ & $2 / 835.50$ & $3 / 761.56$ & $2.3 / 834.94$ & $2 / 836.27$ & 19.45 & $2 / 827.89$ \\
\hline rc101 & $16 / 1731.05$ & $16 / 1726.91$ & $16 / 1735.03$ & $16 / 1731.07$ & $16.0 / 1723.79$ & $16 / 1723.79$ & 13.02 & $16 / 1726.91$ \\
\hline rc102 & $15 / 1554.65$ & $14 / 1659.53$ & $15 / 1557.47$ & $15 / 1551.69$ & $15.0 / 1553.15$ & $15 / 1551.28$ & 14.57 & $14 / 1659.53$ \\
\hline rc103 & $13 / 1353.58$ & $13 / 1369.39$ & $13 / 1351.42$ & $13 / 1351.73$ & $13.0 / 1350.98$ & $13 / 1350.55$ & 14.86 & $13 / 1350.09$ \\
\hline rc104 & $11 / 1249.25$ & $11 / 1229.82$ & $11 / 1229.21$ & $11 / 1232.45$ & $11.3 / 1235.85$ & $11 / 1230.92$ & 13.46 & $11 / 1227.25$ \\
\hline rc105 & $14 / 1483.42$ & $14 / 1478.70$ & $14 / 1484.46$ & $14 / 1473.24$ & $14.0 / 1476.84$ & $14 / 1473.24$ & 12.13 & $14 / 1475.31$ \\
\hline rc106 & $13 / 1440.20$ & $13 / 1436.61$ & $13 / 1439.05$ & $14 / 1414.99$ & $13.0 / 1433.67$ & $\overline{13 / 1423.27}$ & 9.81 & $13 / 1427.21$ \\
\hline $\mathrm{rc} 107$ & $12 / 1275.89$ & $12 / 1283.55$ & $12 / 1276.40$ & $12 / 1283.05$ & $12.0 / 1278.56$ & $\overline{12 / 1274.41}$ & 13.72 & $12 / 1274.89$ \\
\hline rc108 & $11 / 1238.85$ & $11 / 1204.87$ & $11 / 1200.65$ & $11 / 1209.11$ & $11.0 / 1203.61$ & $\frac{1}{11 / 1197.41}$ & 8.92 & $11 / 1197.83$ \\
\hline $\mathrm{rc} 201$ & $4 / 1447.25$ & $4 / 1464.30$ & $4 / 1446.82$ & $4 / 1446.84$ & $4.0 / 1454.23$ & $4 / 1446.03$ & 10.10 & $4 / 1444.94$ \\
\hline rc202 & $3 / 1412.91$ & $3 / 1437.07$ & $3 / 1419.27$ & $3 / 1450.34$ & $3.0 / 1430.52$ & $3 / 1421.34$ & 11.78 & $3 / 1410.74$ \\
\hline rc203 & $3 / 1078.28$ & $3 / 1084.72$ & $3 / 1073.87$ & $3 / 1069.27$ & $3.0 / 1069.15$ & $3 / 1057.16$ & 11.52 & $3 / 1055.19$ \\
\hline rc204 & $3 / 889.25$ & $3 / 902.70$ & $3 / 892.43$ & $3 / 887.45$ & $3.0 / 888.20$ & $3 / 884.72$ & 13.12 & $3 / 884.80$ \\
\hline rc205 & $3 / 1321.70$ & $3 / 1282.58$ & $3 / 1289.76$ & $3 / 1277.60$ & $3.4 / 1236.75$ & $\overline{3 / 1280.33}$ & 12.68 & $3 / 1273.55$ \\
\hline rc206 & $3 / 1191.11$ & $3 / 1218.73$ & $3 / 1191.46$ & $3 / 1207.64$ & $3.0 / 1209.22$ & $3 / 1190.50$ & 11.11 & $3 / 1188.63$ \\
\hline rc207 & $3 / 995.52$ & $3 / 1016.13$ & $3 / 1005.57$ & $3 / 994.48$ & $3.0 / 1002.13$ & $3 / 991.96$ & 12.78 & $3 / 985.03$ \\
\hline rc208 & $3 / 838.07$ & $3 / 847.87$ & $3 / 836.31$ & $3 / 841.34$ & $3.0 / 839.99$ & $3 / 836.29$ & 15.23 & $3 / 836.29$ \\
\hline Time & $15.34 \mathrm{~min}$ & $15.92 \mathrm{~min}$ & 2.78 min* & & & & 11.59 & \\
\hline Gap & $0.23 \% / 0.45 \%$ & $0.45 \% / 1.00 \%$ & $0.23 \% / 0.38 \%$ & $2.27 \% /-0.34 \%$ & $2.05 \% /-0.16 \%$ & $1.36 \% /-0.46 \%$ & & \\
\hline
\end{tabular}

Table 6.7: Comparison with the results for the E-VRPTW of Schneider et al. (2014). SSG: Schneider et al. (2014); HPH: Hiermann et al. (2014); GS: Goeke \& Schneider (2015); KÇ: Keskin \& Çatay (2016). The best values are indicated in boldface (also underlined for new best values).

12.26 min (Keskin \& Çatay, 2016) are reported. Goeke \& Schneider (2015) reported an average CPU time of $2.78 \mathrm{~min}$, but as noted by Keskin \& Çatay (2016), their algorithm uses a priori information on the number of vehicles, which makes a meaningful comparison difficult.

For the E-VRPTWPR, the average CPU time is 11.29 minutes compared to 16.77 minutes as reported 


\begin{tabular}{|c|c|c|c|c|c|}
\hline Name & $\begin{array}{c}\text { KÇ } \\
\text { Best } 10\end{array}$ & Avg 10 & $\begin{array}{l}\text { HGA } \\
\quad \text { Best } 10\end{array}$ & $\mathrm{~T}(\min )$ & BKS \\
\hline c101 & $12.0 / 1051.23$ & $12.0 / 1044.511$ & $12.0 / 1044.511$ & 3.44 & $12.0 / 1051.23$ \\
\hline c102 & $11.0 / 1034.24$ & $\overline{11.0 / 1033.795}$ & $\overline{11.0 / 1033.795}$ & 4.42 & $11.0 / 1034.24$ \\
\hline c103 & $10.0 / 973.39$ & $10.0 / 1001.127$ & $\overline{10.0 / 1001.127}$ & 5.78 & $10.0 / 973.39$ \\
\hline c104 & $10.0 / 886.72$ & $10.0 / 893.0406$ & $10.0 / 893.0406$ & 6.96 & $10.0 / 886.72$ \\
\hline c105 & $11.0 / 1037.78$ & $11.0 / 1052.948$ & $11.0 / 1052.948$ & 3.78 & $11.0 / 1037.78$ \\
\hline c106 & $11.0 / 1024.18$ & $11.0 / 1043.503$ & $11.0 / 1043.503$ & 4.70 & $11.0 / 1024.18$ \\
\hline c107 & $10.0 / 1058.11$ & $11.0 / 1013.761$ & $11.0 / 1013.761$ & 4.69 & $10.0 / 1058.11$ \\
\hline c108 & 10.0/1033.5 & $11.0 / 1000.555$ & $11.0 / 1000.555$ & 7.31 & $10.0 / 1033.5$ \\
\hline c109 & $10.0 / 960.03$ & $10.0 / 946.8438$ & $10.0 / 946.8438$ & 5.16 & $10.0 / 960.03$ \\
\hline c201 & $4.0 / 629.95$ & $4.0 / 658.1089$ & $4.0 / 658.1089$ & 4.93 & $4.0 / 629.95$ \\
\hline c202 & $4.0 / 629.95$ & $4.0 / 645.3894$ & $4.0 / 645.3894$ & 6.18 & $4.0 / 629.95$ \\
\hline c203 & $4.0 / 629.95$ & $4.0 / 643.4462$ & $4.0 / 643.4462$ & 7.30 & $4.0 / 629.95$ \\
\hline c204 & $4.0 / 629.95$ & $4.0 / 636.43$ & $4.0 / 636.43$ & 7.60 & $4.0 / 629.95$ \\
\hline c205 & $4.0 / 629.95$ & $4.0 / 638.1712$ & $4.0 / 638.1712$ & 6.46 & $4.0 / 629.95$ \\
\hline c206 & $4.0 / 629.95$ & $4.0 / 635.3832$ & $4.0 / 635.3832$ & 5.73 & $4.0 / 629.95$ \\
\hline c207 & $4.0 / 629.95$ & $4.0 / 632.7976$ & $4.0 / 632.7976$ & 6.27 & $4.0 / 629.95$ \\
\hline c208 & $4.0 / 629.95$ & $4.0 / 638.1712$ & $4.0 / 638.1712$ & 6.41 & $4.0 / 629.95$ \\
\hline r101 & $18.0 / 1661.33$ & $18.0 / 1630.135$ & $18.0 / 1630.135$ & 10.36 & $18.0 / 1661.33$ \\
\hline r102 & $16.0 / 1461.48$ & $15.0 / 1521.325$ & $\overline{15.0 / 1521.325}$ & 10.26 & $16.0 / 1461.48$ \\
\hline r103 & $13.0 / 1262.75$ & $13.0 / 1264.807$ & $\overline{13.0 / 1264.807}$ & 7.77 & $13.0 / 1262.75$ \\
\hline r104 & $11.0 / 1078.99$ & $11.0 / 1089.919$ & $11.0 / 1089.919$ & 12.87 & $11.0 / 1078.99$ \\
\hline r105 & $15.0 / 1373.94$ & $14.0 / 1396.798$ & 14.0/1396.798 & 9.57 & $15.0 / 1373.94$ \\
\hline r106 & $13.0 / 1310.46$ & $13.0 / 1281.087$ & $13.0 / 1281.087$ & 9.69 & $13.0 / 1310.46$ \\
\hline r107 & $12.0 / 1118.91$ & $12.0 / 1127.709$ & $\overline{12.0 / 1127.709}$ & 15.67 & $12.0 / 1118.91$ \\
\hline r108 & $11.0 / 1031.14$ & $11.0 / 1042.797$ & $11.0 / 1042.797$ & 17.69 & $11.0 / 1031.14$ \\
\hline r109 & $13.0 / 1201.04$ & $12.0 / 1265.818$ & $12.0 / 1265.818$ & 15.04 & $13.0 / 1201.04$ \\
\hline r110 & $11.0 / 1112.8$ & $11.0 / 1094.995$ & $11.0 / 1094.995$ & 11.31 & $11.0 / 1112.8$ \\
\hline r111 & $12.0 / 1084.13$ & $11.0 / 1147.225$ & $11.0 / 1147.225$ & 17.95 & $12.0 / 1084.13$ \\
\hline r112 & $11.0 / 1017.31$ & $11.0 / 1013.945$ & $\overline{11.0 / 1013.945}$ & 7.68 & $11.0 / 1017.31$ \\
\hline r201 & $3.0 / 1266.06$ & $3.0 / 1261.637$ & $3.0 / 1261.637$ & 8.41 & $3.0 / 1258.39$ \\
\hline r202 & $3.0 / 1052.32$ & $3.0 / 1051.457$ & $3.0 / 1051.457$ & 9.93 & $3.0 / 1052.32$ \\
\hline r203 & $3.0 / 895.54$ & $3.0 / 900.6014$ & $\overline{3.0 / 900.6014}$ & 10.79 & $3.0 / 895.54$ \\
\hline r204 & $2.0 / 780.14$ & $2.0 / 783.5278$ & $2.0 / 783.5278$ & 15.76 & $2.0 / 780.14$ \\
\hline r205 & $3.0 / 987.36$ & $3.0 / 987.362$ & $3.0 / 987.362$ & 9.47 & $3.0 / 987.36$ \\
\hline r206 & $3.0 / 922.7$ & $3.0 / 924.4825$ & $3.0 / 924.4825$ & 11.25 & $3.0 / 922.7$ \\
\hline r207 & $2.0 / 846.59$ & $2.0 / 846.5337$ & $2.0 / 846.5337$ & 15.69 & $2.0 / 846.59$ \\
\hline r208 & $2.0 / 736.12$ & $2.0 / 736.6421$ & $\overline{2.0 / 736.6421}$ & 16.08 & $2.0 / 736.12$ \\
\hline r209 & $3.0 / 868.95$ & $3.0 / 867.7957$ & $3.0 / 867.7957$ & 10.83 & $3.0 / 868.95$ \\
\hline r 210 & $3.0 / 843.36$ & $3.0 / 845.2673$ & $\overline{3.0 / 845.2673}$ & 10.72 & $3.0 / 843.36$ \\
\hline r211 & $2.0 / 862.56$ & $2.0 / 857.1001$ & $2.0 / 857.1001$ & 19.61 & $2.0 / 862.56$ \\
\hline rc101 & $16.0 / 1684.84$ & $15.0 / 1725.727$ & $\overline{15.0 / 1725.727}$ & 7.01 & $16.0 / 1684.84$ \\
\hline rc102 & $14.0 / 1555.9$ & $14.0 / 1540.257$ & $14.0 / 1540.257$ & 9.26 & $14.0 / 1555.9$ \\
\hline rc103 & $13.0 / 1329.58$ & $12.0 / 1388.715$ & $\overline{12.0 / 1388.715}$ & 8.99 & $13.0 / 1329.58$ \\
\hline rc104 & $11.0 / 1202.93$ & $11.0 / 1181.263$ & $11.0 / 1181.263$ & 12.62 & $11.0 / 1202.93$ \\
\hline rc105 & $14.0 / 1458.49$ & $14.0 / 1463.486$ & $\overline{14.0 / 1463.486}$ & 14.10 & $14.0 / 1458.49$ \\
\hline rc106 & $13.0 / 1422.96$ & $13.0 / 1397.55$ & $13.0 / 1397.55$ & 12.25 & $13.0 / 1422.96$ \\
\hline rc107 & $12.0 / 1261.03$ & $12.0 / 1255.031$ & $\overline{12.0 / 1255.031}$ & 14.99 & $12.0 / 1261.03$ \\
\hline rc108 & $11.0 / 1185.68$ & $11.0 / 1165.596$ & $\overline{11.0 / 1165.596}$ & 11.48 & $11.0 / 1185.68$ \\
\hline rc201 & $4.0 / 1446.84$ & $4.0 / 1446.032$ & $4.0 / 1446.032$ & 7.04 & $4.0 / 1446.84$ \\
\hline rc202 & $3.0 / 1416.96$ & $3.0 / 1434.175$ & $\overline{3.0 / 1434.175}$ & 11.08 & $3.0 / 1416.96$ \\
\hline rc203 & $3.0 / 1069.27$ & $3.0 / 1061.117$ & $3.0 / 1061.117$ & 10.21 & $3.0 / 1069.27$ \\
\hline rc204 & $3.0 / 887.76$ & $3.0 / 887.1029$ & $3.0 / 887.1029$ & 12.19 & $3.0 / 887.76$ \\
\hline rc205 & $3.0 / 1262.22$ & $3.0 / 1289.078$ & $\overline{3.0 / 1289.078}$ & 10.24 & $3.0 / 1262.22$ \\
\hline rc206 & $3.0 / 1213.89$ & $3.0 / 1200.743$ & $3.0 / 1200.743$ & 9.89 & $3.0 / 1213.89$ \\
\hline rc207 & $3.0 / 993.49$ & $3.0 / 985.6748$ & $\overline{3.0 / 985.6748}$ & 11.83 & $3.0 / 993.49$ \\
\hline rc208 & $3.0 / 839.71$ & $3.0 / 836.9279$ & $\overline{3.0 / 836.9279}$ & 13.33 & $3.0 / 839.71$ \\
\hline $\begin{array}{l}\text { Time } \\
\text { Gap }\end{array}$ & $\begin{array}{c}16.77 \mathrm{~min} \\
0.0 \% / 0.01 \%\end{array}$ & $0.5 \% / 0.20 \%$ & $-0.9 \% / 0.43 \%$ & 9.96 & \\
\hline
\end{tabular}

Table 6.8: Comparison with the results for the E-VRPTWPR of Keskin \& Çatay (2016). The best values are indicated in boldface (also underlined for new best values).

by Keskin \& Çatay (2016). We found 28 new best solutions and six solutions that reduced the number of vehicles. Most improvements occurred in the $\mathrm{r}$ and $\mathrm{rc}$ variants, in both the type- 1 and type- 2 categories. 


\subsection{Performance Analysis - E-FSMFTW}

The E-FSMFTW is a closely related problem that considers a heterogeneous fleet of pure-electric vehicles and uses a fixed recharging scheme (always to maximum capacity). The instances are based on the previously mentioned E-VRPTW instances (Schneider et al., 2014) combined with the vehicle type definition for the FSMF (Liu \& Shen, 1999). However, this type definition is extended by a varying battery capacity but a shared consumption rate.

\begin{tabular}{ll|rrr|rrr|rrr}
\hline & & \multicolumn{3}{c}{ HPRH } & & \multicolumn{3}{c}{ Montoya } & \multicolumn{2}{c}{ HGA } \\
Type & & Best 10 & Avg 10 & $\mathrm{T}(\min )$ & Best 10 & Avg 10 & $\mathrm{T}(\mathrm{min})$ & Best 10 & Avg 10 & $\mathrm{T}(\mathrm{min})$ \\
\hline $\mathrm{A}$ & $\mathrm{c}$ & 6470.08 & 6487.28 & 29.37 & 6464.93 & 6477.48 & 28.48 & $\mathbf{6 4 5 8 . 6 4}$ & $\mathbf{6 4 6 2 . 4 7}$ & $\mathbf{1 9 . 6 2}$ \\
& $\mathrm{r}$ & 3646.66 & 3673.97 & 30.05 & 3660.94 & 3676.95 & 21.95 & $\mathbf{3 6 1 6 . 2 8}$ & $\mathbf{3 6 2 4 . 9 9}$ & $\mathbf{2 0 . 3 4}$ \\
& $\mathrm{rc}$ & 4600.30 & 4639.24 & $\mathbf{1 5 . 4 8}$ & 4602.54 & 4621.68 & 27.67 & $\mathbf{4 5 7 9 . 8 4}$ & $\mathbf{4 5 9 1 . 8 6}$ & 17.92 \\
\hline $\mathrm{B}$ & $\mathrm{c}$ & 2100.85 & 2114.16 & 25.21 & 2102.16 & 2109.79 & 25.85 & $\mathbf{2 0 9 7 . 4 3}$ & $\mathbf{2 0 9 8 . 8 3}$ & $\mathbf{1 7 . 1 6}$ \\
& $\mathrm{r}$ & 1623.81 & 1643.92 & 22.02 & 1658.11 & 1667.44 & 21.55 & $\mathbf{1 6 1 7 . 0 4}$ & $\mathbf{1 6 2 7 . 2 6}$ & $\mathbf{1 5 . 2 3}$ \\
& $\mathrm{rc}$ & 1933.33 & 1955.53 & 16.72 & 1966.50 & 1997.52 & 18.41 & $\mathbf{1 9 2 4 . 0 3}$ & $\mathbf{1 9 3 5 . 0 8}$ & $\mathbf{1 2 . 5 4}$ \\
\hline $\mathrm{C}$ & $\mathrm{c}$ & 1495.12 & 1503.53 & 22.86 & 1498.40 & 1505.52 & 19.57 & $\mathbf{1 4 9 3 . 6 9}$ & $\mathbf{1 4 9 4 . 5 3}$ & $\mathbf{1 2 . 4 7}$ \\
& $\mathrm{r}$ & 1349.89 & 1368.57 & 21.92 & 1382.08 & 1391.79 & 34.42 & $\mathbf{1 3 4 4 . 2 6}$ & $\mathbf{1 3 5 2 . 6 9}$ & $\mathbf{1 4 . 7 6}$ \\
& $\mathrm{rc}$ & 1576.43 & 1594.96 & 17.26 & 1599.57 & 1612.44 & 28.02 & $\mathbf{1 5 7 0 . 5 5}$ & $\mathbf{1 5 7 9 . 0 3}$ & $\mathbf{1 3 . 1 6}$ \\
\hline
\end{tabular}

Table 6.9: Comparison with heuristics from the literature for the E-FSMFTW. HPRH: Hiermann et al. (2016); Montoya (2016)

Table 6.9 shows the results for the E-FSMF benchmark instances. The results are grouped by vehicle type (A,B,C), and we report the average values for each instance type (c,r,rc). For the ALNS of Hiermann et al. (2016) (HPRH), the parallel matheuristic of Montoya (2016), and our HGA, we report (a) the best objective value, (b) the average over 10 runs, and (c) the average CPU time in minutes. HGA performs very well compared to the other algorithms, finding better results on average for all instance-type combination. The time over all instances is 15.80 min for the HGA, which compares well with $22.66 \mathrm{~min}$ (HPRH) and 25.20 min (Montoya).

Detailed results are presented in the Appendix. Our algorithm found 119 new best solutions for the 168 instances in the benchmark set.

\section{Conclusions}

In this paper, we have introduced a vehicle routing problem variant considering a mix of conventional, hybrid, and electric vehicles. The study of this prototypical problem allows to better understand how to compose and route a fleet of vehicles with different properties and different constraints, and to study the relative role of each vehicle in operational settings. This problem bridges the gap between the classical VRP and the electric VRP, and includes possible plug-in hybrid vehicles which form an intermediate option between the two propulsion modes.

To solve this problem, we have introduced an efficient hybrid genetic algorithm. We implemented a layered route evaluation approach to solve the subproblems, therefore decomposing the problem into a pure assignment and sequencing problem, in the top layer, and problem-specific route evaluations in a bottom layer. We use labeling and greedy evaluation methods to insert recharging stations visits and select the propulsion mode over the trip for hybrid vehicles. The performance of our algorithm is demonstrated on a variety of benchmark instances of the related E-FSMF and the E-VRPTW variants. As illustrated by our computational results, the method produced average solutions of equal or higher quality than existing algorithms for these problems. It also found 119 new best solutions for the E-FSMF, 11 for the E-VRPTW and 19 for the E-VRPTWPR.

Our computational studies also show the relevance of this broader problem formulation and provide insights into various managerial implications. In particular, we considered different fuel and energy price scenarios to observe their impact on the optimized fleet mix. Our results show that the operational cost of the best mixed fleet can be $7 \%$ lower than the best homogeneous fleet with either ICEV, BEV or PHEV. Therefore, logistic activities in the coming decades may require a mix of vehicles and propulsion types to 
achieve competitiveness. Furthermore, even if hybrid plug-in vehicles tend to consume more energy than fully electric vehicles due to their heavier base load, our experiments confirm that their ability to switch fuel on a trip can help cutting down operational costs. PHEV do not usually represent the majority of the vehicles in our optimized solutions, but a few hybrid vehicles are recurrent in a majority of scenarios with intermediate fuel and energy price.

To stimulate further research, we make available a new benchmark set based on the classical electric VRP and vehicle parameters from real-world data. ${ }^{1}$ As a next step we will add additional real-world constraints in the form of city-center restrictions. These restrictions prohibit or penalize the use of ICEVs in specified areas, as is the case in several cities around the world. This will provide an insight into the fleet needed for cost-efficient operation in urban areas. We will also further explore the systematic evaluation methodology and its use for other VRP classes.

\section{Acknowledgments}

We wish to thank our colleagues at the Austrian Institute of Technology and the University of Vienna as well as the participants of various conferences for useful discussions and suggestions. This work is partially funded by the Austrian Climate and Energy Fund within the Electric Mobility Flagship Projects program under grant 834868 (project VECEPT). This support is gratefully acknowledged. Furthermore, we like to mention that a substantial part of Gerhard Hiermanns work has been done while he was affiliated with the Austrian Institute of Technology

\section{References}

Abdallah, T. (2013). The Plug-In Hybrid Electric Vehicle Routing Problem with Time Windows. Master's thesis University of Waterloo Canada.

AustriaTech (2014). Annex: Electric fleets in urban logistics, project ENCLOSE. Last accessed 2018-03-08.

Baldacci, R., Battarra, M., \& Vigo, D. (2008). Routing a heterogeneous fleet of vehicles. In B. Golden, S. Raghavan, \& E. Wasil (Eds.), The Vehicle Routing Problem: Latest Advances and New Challenges (pp. 3-27). New York, NY, USA: Springer US volume 43 of Operations Research/Computer Science Interfaces.

Çatay, B., \& Keskin, M. (2017). The impact of quick charging stations on the route planning of electric vehicles. In 2017 IEEE Symposium on Computers and Communications (ISCC) (pp. 152-157). Heraklion, Greece.

Christiaens, J., \& Vanden Berghe, G. (2016). A fresh ruin \& recreate implementation for the capacitated vehicle routing problem. Technical Report KU Leuven Ghent, Belgium.

Conrad, R. G., \& Figliozzi, M. A. (2011). The recharging vehicle routing problem. In T. Doolen, \& E. Van Aken (Eds.), Proceedings of the 2011 Industrial Engineering Research Conference. Reno, NV, USA.

Desaulniers, G., Errico, F., Irnich, S., \& Schneider, M. (2016). Exact algorithms for electric vehicle-routing problems with time windows. Operations Research, 64, 1388-1405.

Edenhofer, O., Pichs-Madruga, R., Sokona, Y., Minx, J. C., Farahani, E., Kadner, K., S. Seyboth, Adler, A., Baum, I., Brunner, S., Eickemeier, P., Kriemann, B., Savolainen, J., Schlömer, S., Von Stechow, C., \& Zwickel, T. (Eds.) (2014). Climate Change 2014: Mitigation of Climate Change. Working Group III Contribution to the Fifth Assessment Report of the Intergovernmental Panel on Climate Change. New York, NY, USA: Cambridge University Press.

\footnotetext{
${ }^{1}$ http://www.vrp-rep.org/datasets/item/2017-0029.html
} 
Erdoğan, S., \& Miller-Hooks, E. (2012). A green vehicle routing problem. Transportation Research Part E: Logistics and Transportation Review, 48, 100-114.

Felipe, A., Ortuño, M. T., Righini, G., \& Tirado, G. (2014). A heuristic approach for the green vehicle routing problem with multiple technologies and partial recharges. Transportation Research Part E: Logistics and Transportation Review, 71, 111-128.

Garaix, T., Artigues, C., Feillet, D., \& Josselin, D. (2010). Vehicle routing problems with alternative paths: An application to on-demand transportation. European Journal of Operational Research, 204, 62-75.

Goeke, D., \& Schneider, M. (2015). Routing a mixed fleet of electric and conventional vehicles. European Journal of Operational Research, 245, 81-99.

Goel, A., \& Vidal, T. (2014). Hours of service regulations in road freight transport: An optimization-based international assessment. Transportation Science, 48, 391-412.

Hiermann, G., Puchinger, J., \& Hartl, R. F. (2014). The electric fleet size and mix vehicle routing problem with time windows and recharging stations. Working paper March 2014, University of Vienna, Austria.

Hiermann, G., Puchinger, J., Ropke, S., \& Hartl, R. F. (2016). The electric fleet size and mix vehicle routing problem with time windows and recharging stations. European Journal of Operational Research, 252, 995-1018.

Irnich, S., \& Desaulniers, G. (2005). Shortest path problems with resource constraints. In G. Desaulniers, J. Desrosiers, \& M. M. Solomon (Eds.), Column Generation (pp. 33-65). New York, NY, USA: Springer US.

Irnich, S., \& Villeneuve, D. (2006). The shortest-path problem with resource constraints and k-cycle elimination for $\mathrm{k} \geq 3$. INFORMS Journal on Computing, 18, 391-406.

Juan, A. A., Mendez, C. A., Faulin, J., Armas, J., \& Grasman, S. E. (2016). Electric vehicles in logistics and transportation: A survey on emerging environmental, strategic, and operational challenges. Energies, 9,86 .

Keskin, M., \& Çatay, B. (2016). Partial recharge strategies for the electric vehicle routing problem with time windows. Transportation Research Part C: Emerging Technologies, 65, 111-127.

Lebeau, P., De Cauwer, C., Van Mierlo, J., Macharis, C., Verbeke, W., \& Coosemans, T. (2015). Conventional, hybrid, or electric vehicles: Which technology for an urban distribution centre? The Scientific World Journal, 2015.

Liu, F.-H., \& Shen, S.-Y. (1999). The fleet size and mix vehicle routing problem with time windows. Journal of the Operational Research Society, 50, 721-732.

Montoya, J.-A. (2016). Electric Vehicle Routing Problems: Models and solution approaches. Ph.D. thesis Université d'Angers France.

Nagata, Y., Bräysy, O., \& Dullaert, W. (2010). A penalty-based edge assembly memetic algorithm for the vehicle routing problem with time windows. Computers $\& 3$ Operations Research, 37, 724-737.

Pelletier, S., Jabali, O., \& Laporte, G. (2016). Goods distribution with electric vehicles: Review and research perspectives (50th anniversary invited article). Transportation Science, 50, 3-22.

Plötz, P., Gnann, T., Wietschel, M., \& Kühn, A. (2013). Markthochlaufszenarien für Elektrofahrzeuge Langfassung, Fraunhofer ISI, Karlsruhe. Last accessed 2018-03-08.

Prins, C. (2004). A simple and effective evolutionary algorithm for the vehicle routing problem. Computers 83 Operations Research, 31, 1985-2002. 
Prins, C. (2009). Two memetic algorithms for heterogeneous fleet vehicle routing problems. Engineering Applications of Artificial Intelligence, 33, 916-928.

Ropke, S., \& Pisinger, D. (2006). An adaptive large neighborhood search heuristic for the pickup and delivery problem with time windows. Transportation Science, 40, 455-472.

Sassi, O., Cherif-Khettaf, W. R., \& Oulamara, A. (2015). Vehicle routing problem with mixed fleet of conventional and heterogenous electric vehicles and time dependent charging costs. International Journal of Mathematical, Computational, Physical, Electrical and Computer Engineering, 9, 171-181.

Schneider, M., Stenger, A., \& Goeke, D. (2014). The electric vehicle routing problem with time windows and recharging stations. Transportation Science, 48, 500-520.

Subramanian, A., Uchoa, E., \& Ochi, L. S. (2013). A hybrid algorithm for a class of vehicle routing problems. Computers $\& 3$ Operations Research, 40, 2519-2531.

Toth, P., \& Vigo, D. (2014). Vehicle Routing: Problems, Methods, and Applications, Second Edition. Philadelphia, PA, USA: Society for Industrial and Applied Mathematics.

Vidal, T. (2017). Node, edge, arc routing and turn penalties : Multiple problems - One neighborhood extension. Operations Research, 65, 992-1010.

Vidal, T., Crainic, T. G., Gendreau, M., Lahrichi, N., \& Rei, W. (2012). A hybrid genetic algorithm for multidepot and periodic vehicle routing problems. Operations Research, 60, 611-624.

Vidal, T., Crainic, T. G., Gendreau, M., \& Prins, C. (2013). A hybrid genetic algorithm with adaptive diversity management for a large class of vehicle routing problems with time-windows. Computers \& Operations Research, 40, 475-489.

Vidal, T., Crainic, T. G., Gendreau, M., \& Prins, C. (2015a). Time-window relaxations in vehicle routing heuristics. Journal of Heuristics, 21, 329-358.

Vidal, T., Crainic, T. G., Gendreau, M., \& Prins, C. (2015b). Timing problems and algorithms: Time decisions for sequences of activities. Networks, 65, 102-128.

Zündorf, T. (2014). Electric Vehicle Routing with Realistic Recharging Models. Master's thesis Karlsruhe Institute of Technology Germany. 


\section{Appendix A. Appendix}

\section{Appendix A.1. Greedy charging policy for PHEVs}

In Section 4.1, we described the extension functions for evaluating the routes of PHEVs. We outlined the key steps of the greedy policy required to ensure that the cost of the route is minimal without violating the time and energy constraints. In summary, these are: 1) use electric energy mode first, since we assume that electricity is cheaper than fossil fuel; 2-3) recharge only when necessary or to avoid waiting time, to minimize costs while ensuring time-feasibility similar to that for BEVs; and 4) revert recharging operations only if time windows are violated, to ensure that fossil fuel is used only when necessary.

As for the BEV extension function, we define the following resource extension functions, denoted $T_{i}^{\text {res }}$. The intermediate calculations, starting with $\Delta$, are described along with the functions.

$$
\begin{aligned}
T_{j}^{\mathrm{DIST}} & =T_{i}^{\mathrm{DIST}}+d_{i j} \\
T_{j}^{\mathrm{Q}} & =T_{i}^{\mathrm{Q}}+q_{j}
\end{aligned}
$$

The distance and capacity resources are $T_{i}^{\text {DIST }}$ and $T_{i}^{Q}$. Their respective extensions simply add the distance traveled or the additional load.

$$
\begin{aligned}
T_{j}^{\mathrm{DUR}^{\mathrm{F}}} & =T_{i}^{\mathrm{DUR}^{\mathrm{F}}}+t_{i j}+s_{j}+\Delta^{\mathrm{WT}^{\mathrm{F}}} \\
T_{j}^{\mathrm{TW}} & =T_{i}^{\mathrm{TW}}+\Delta^{\mathrm{TW}^{\mathrm{F}}} \\
\Delta^{\mathrm{DUR}^{\mathrm{F}}} & =T_{i}^{\mathrm{DUR}^{\mathrm{F}}}-T_{i}^{\mathrm{TW}}+t_{i j} \\
\Delta^{\mathrm{WT}^{\mathrm{F}}} & =\max \left\{e_{j}-\Delta^{\mathrm{DUR}^{\mathrm{F}}}-e_{0}, 0\right\} \\
\Delta^{\mathrm{TW}^{\mathrm{F}}} & =\max \left\{e_{0}+\Delta^{\mathrm{DUR}^{\mathrm{F}}}-l_{j}, 0\right\}
\end{aligned}
$$

According to our formulation, PHEVs can use either the electric engine or the ICE. The use of the electric energy stored in the battery may require a reloading operation, which takes time. This could lead to a violation of the time constraint. Equation (A.3) calculates the duration of the route if only the ICE is used; this is the minimal duration for this route. Time-window violations can be repaired only by reverting a recharging operation to this lower bound. Any further violation is penalized using a time warp, as described in Section 3.1.1 for BEVs. Using the intermediate calculations (A.5)-(A.7), the additional time warp is computed in Equation (A.4).

$$
\begin{aligned}
T_{j}^{\mathrm{DUR}} & =T_{i}^{\mathrm{DUR}}+t_{i j}+s_{j}+\Delta^{\mathrm{WT}}+\vec{\Delta}^{\mathrm{T}^{\mathrm{RC}}}-\Delta^{\mathrm{T}^{\mathrm{ADJ}}} \\
T_{j}^{\mathrm{DIST}^{\mathrm{F}}} & =T_{i}^{\mathrm{DIST} \mathrm{T}^{\mathrm{F}}}+\left(\Delta^{\mathrm{EY} \mathrm{Y}^{\mathrm{RC}}}+\Delta^{\mathrm{T}^{\mathrm{ADJ}}} / g\right) / r_{\mathrm{E}} \\
\vec{\Delta}^{\mathrm{Y}^{\mathrm{RC}}} & =\max \left\{\min \left\{d_{i j} \cdot r_{\mathrm{E}}-T_{i}^{\mathrm{Y}}, T_{i}^{\mathrm{YAR}}, T_{i}^{\mathrm{TAR}} / g\right\}, 0\right\} \\
\vec{\Delta}^{\mathrm{T}^{\mathrm{RC}}} & =\vec{\Delta}^{\mathrm{Y} \mathrm{RC}} \cdot g \\
\Delta & =T_{i}^{\mathrm{DUR}}+\vec{\Delta}^{\mathrm{Y}^{\mathrm{RC}}} \cdot g-T_{i}^{\mathrm{TW}}+t_{i j} \\
\Delta^{\mathrm{EY}} & =\max \left\{d_{i j} \cdot r_{\mathrm{E}}-T_{i}^{\mathrm{Y}}-\vec{\Delta}^{\mathrm{Y}^{\mathrm{RC}}}, 0\right\} \\
\Delta^{\mathrm{TW}} & =\max \left\{e_{0}+\Delta-l_{j}, 0\right\} \\
\Delta^{\mathrm{T}^{\mathrm{ADJ}}} & =\max \left\{\Delta^{\mathrm{TW}}-\Delta^{\mathrm{TW}^{\mathrm{F}}}, 0\right\} \\
\Delta^{\mathrm{WT}} & =\max \left\{e_{j}-\left(\Delta-\Delta^{\mathrm{T}^{\mathrm{ADJ}}}\right)-e_{0}, 0\right\}
\end{aligned}
$$

Equation (A.8) calculates the duration of the route after vertex $j$ based on the travel time $\left(t_{i j}\right)$, service time $\left(s_{j}\right)$, waiting time $\left(\Delta^{\mathrm{WT}}\right)$, and PHEV-specific calculations. These consist of two terms: a) the recharging time at the last recharging station visited before vertex $j\left(\vec{\Delta}^{\mathrm{RC}}\right)$, and b) the time gained by using fuel instead of electric energy $\left(\Delta^{\mathrm{T}^{\mathrm{ADS}}}\right)$. Similarly to the BEV case, we recharge only when necessary. However, we can avoid 
time-window violations by choosing not to recharge. Equation (A.10) uses the minimum energy required to recharge, the amount available to recharge based on the energy restriction, and the time available. If the amount of electric energy required does not exceed the current energy level, the first term will be negative and the equation evaluates to zero. The duration until arrival at vertex $j$ is calculated in Equation (A.12), which adds the time required to recharge $\left(\vec{\Delta}^{\mathrm{Y}^{\mathrm{RC}}} \cdot g\right)$ to the travel time minus the previous time warp. With this, we can calculate the lateness and thus the time warp required when recharging (A.14). However, as stated previously, we can avoid time warp by using fuel instead of electric energy, and our policy enforces this behavior. Equation (A.15) is used to calculate the repairable time warp, which is the difference between the time warp with and without recharging operations. This value has to be removed from the duration when calculating the waiting time (see A.16).

The second equation in this listing (A.9) calculates the distance traveled using fuel instead of electric energy. This value is increased by summing the energy that is not rechargeable $\left(\Delta^{\mathrm{EY}}\right)$ and the electric energy traded for fuel to avoid time warp $\left(\Delta^{\mathrm{T}^{\mathrm{ADJ}}} / g\right)$ divided by the electric energy consumption $\left(r_{\mathrm{E}}\right)$ to get the distance value.

$$
\begin{aligned}
T_{j}^{\mathrm{YAR}} & = \begin{cases}Y-\Delta^{\mathrm{Y}^{\mathrm{RC}}} & \text { if } j \in \mathcal{F} \\
\Delta^{\mathrm{YAR}} & \text { otherwise }\end{cases} \\
T_{j}^{\mathrm{TAR}} & =\min \left\{\max \left\{0, l_{j}-e_{0}-T_{j}^{\mathrm{DUR}}+T_{j}^{\mathrm{TW}}\right\}, \Delta^{\mathrm{TAR}}\right\} \\
T_{j}^{\mathrm{Y}} & =T_{i}^{\mathrm{Y}}-d_{i j} \cdot r_{\mathrm{E}}+\Delta^{\mathrm{Y}^{\mathrm{RC}}} \\
\Delta^{\mathrm{YAR}} & = \begin{cases}0 & \text { if } \Delta^{\mathrm{A}^{\mathrm{ADJ}}}>0 \\
T_{i}^{\mathrm{YAR}}-\vec{\Delta}^{\mathrm{Y}^{\mathrm{RC}}} & \text { otherwise }\end{cases} \\
\Delta^{\mathrm{TAR}} & = \begin{cases}+\infty & \text { if } j \in \mathcal{F} \\
T_{i}^{\mathrm{TAR}}-\vec{\Delta}^{\mathrm{Y}} & \text { otherwise } \\
\Delta^{\mathrm{Y}^{\mathrm{RC}}} & =\vec{\Delta}^{\mathrm{Y}^{\mathrm{RC}}}+\min \left\{\Delta^{\mathrm{WT}} / g, \Delta^{\mathrm{YAR}}\right\}\end{cases}
\end{aligned}
$$

To keep track of the amount of energy we can recharge, we use two resources: the amount of rechargeable energy $\left(T^{\mathrm{YAR}}\right)$ and the time available to recharge $\left(T^{\mathrm{TAR}}\right)$. The first value is updated by calculating the total amount of energy recharged to this point (A.22). When we extend to a recharging station, the previous state plus the amount recharged define the value. Otherwise, we have to differentiate between the two cases described in (A.20): a) time adjustment $\left(\Delta^{\mathrm{ADJ}}>0\right)$, which will set the rechargeable amount to zero because we have to reverse earlier recharging decisions, so no additional recharging is viable; and b) no adjustment, so we reduce the possible amount by the amount we have already recharged.

The second resource (time available to recharge) is updated using Equation (A.18). This finds the minimum of a) the maximum, non-negative available time in the vertex and b) either the previous available time minus the recharging time for the current vertex $\left(\vec{\Delta}^{\mathrm{PC}}\right)$, or infinity if the current vertex is a recharging station.

Using these extension functions we can define the objective function as follows:

$$
T_{j}^{\text {cost }}=T_{j}^{\mathrm{DIST}} \cdot r_{\mathrm{E}} \cdot c_{\mathrm{E}}+T_{j}^{\mathrm{DIST}{ }^{\mathrm{F}}} \cdot r_{\mathrm{F}} \cdot c_{\mathrm{F}}
$$

\section{Appendix A.2. Labeling for PHEVs}

The resources for the PHEV labeling are:

$$
R^{\mathrm{PHEV}}=\left\{v, T^{\mathrm{COST}}, T^{\mathrm{DUR}}, T^{\mathrm{TW}}, T^{\mathrm{DUR}}, T^{\mathrm{Y}}, T^{\mathrm{YAR}}, T^{\mathrm{TAR}}\right\}
$$

where $T^{\mathrm{COST}}(L)$ is the cost of the label, $T^{\mathrm{DUR}}(L)$ the time duration at $v(L), T^{\mathrm{DUR}^{\mathrm{F}}}(L)$ time duration without recharging, $T^{\mathrm{TW}}\left(L_{1}\right)$ the time warp, and $T^{\mathrm{Y}}(L)$ the current energy level. $T^{\mathrm{YAR}}(L)$ is the rechargeable energy 
and $T^{\mathrm{TAR}}(L)$ the maximum recharging time, both at the last recharging station visited. These values are taken directly from the result of the evaluation methods described above.

$$
\begin{aligned}
T^{\mathrm{COST}}\left(L_{1}\right)+\rho^{\mathrm{TW}} \cdot T^{\mathrm{TW}}\left(L_{1}\right) & \leq T^{\mathrm{COST}}\left(L_{2}\right)+\rho^{\mathrm{TW}} \cdot T^{\mathrm{TW}}\left(L_{2}\right) \\
T^{\mathrm{DUR}}\left(L_{1}\right) & \leq T^{\mathrm{DUR}}\left(L_{2}\right) \\
T^{\mathrm{DUR}}\left(L_{1}\right) & \leq T^{\mathrm{DUR}}\left(L_{2}\right) \\
T^{\mathrm{Y}}\left(L_{1}\right) & \geq T^{\mathrm{Y}}\left(L_{2}\right) \\
T^{\mathrm{Y}}\left(L_{1}\right)+T^{\mathrm{YAR}}\left(L_{1}\right)^{\prime} & \geq T^{\mathrm{Y}}\left(L_{2}\right)+T^{\mathrm{YAR}}\left(L_{2}\right)^{\prime}
\end{aligned}
$$

The dominance criterion is defined as follows: A label $L_{1}$ dominates $L_{2}$ if the cost ( + time warp penalty) (A.24), the duration with recharging (A.25), and the duration without recharging (A.25) are less than the values for $L_{2}$, and the current (A.27) and the maximum available energy (A.28) are greater than the vaues for $L_{2}$.

This simple criterion is similar to that defined for BEVs in Section 3.1.2. It adds a check for the time without recharging, because we could avoid recharging by using the ICE. This prevents the removal of labels that are potentially costly but could fulfill customer time-window restrictions. Clearly, this will increase the number of labels we have to consider and thus impact the overall CPU time. To strengthen the criterion and allow for a more aggressive removal of labels, we define a stricter criterion as follows:

$$
\begin{aligned}
& T^{\mathrm{COST}}\left(L_{1}\right)+\rho^{\mathrm{TW}} \cdot T^{\mathrm{TW}}\left(L_{1}\right) \leq T^{\mathrm{COST}}\left(L_{2}\right)+\rho^{\mathrm{TW}} \cdot T^{\mathrm{TW}}\left(L_{2}\right) \\
& T^{\mathrm{DUR}^{\mathrm{F}}}\left(L_{1}\right) \leq T^{\mathrm{DUR}^{\mathrm{F}}}\left(L_{2}\right) \\
& T^{\mathrm{DUR}}\left(L_{1}\right) \leq T^{\mathrm{DUR}}\left(L_{2}\right) \vee \\
& \left(\begin{array}{c}
\left(T^{\mathrm{DUR}}\left(L_{1}\right)-T^{\mathrm{DUR}^{\mathrm{F}}}\left(L_{1}\right) \geq T_{1-2}^{\mathrm{DUR}} \wedge\right. \\
T^{\mathrm{COST}}\left(L_{1}\right)+\rho^{\mathrm{TW}} \cdot T^{\mathrm{TW}}\left(L_{1}\right)+\psi\left(T_{1-2}^{\mathrm{DUR}} / g\right) \leq T^{\mathrm{COST}}\left(L_{2}\right)+\rho^{\mathrm{TW}} \cdot T^{\mathrm{TW}}\left(L_{2}\right)
\end{array}\right) \\
& T^{\mathrm{Y}}\left(L_{1}\right)+T^{\mathrm{YAR}}\left(L_{1}\right)^{\prime} \geq T^{\mathrm{Y}}\left(L_{2}\right)+T^{\mathrm{YAR}}\left(L_{2}\right)^{\prime} \vee \\
& \left(\begin{array}{c}
\left(T^{\mathrm{DUR}}\left(L_{1}\right)-T^{\mathrm{DUR}^{\mathrm{F}}}\left(L_{1}\right)\right) \cdot g \geq T_{2-1}^{\mathrm{Y}} \wedge \\
T^{\mathrm{COST}}\left(L_{1}\right)+\rho^{\mathrm{TW}} \cdot T^{\mathrm{TW}}\left(L_{1}\right)+\psi\left(T_{2-1}^{\mathrm{Y}}\right) \leq T^{\mathrm{COST}}\left(L_{2}\right)+\rho^{\mathrm{TW}} \cdot T^{\mathrm{TW}}\left(L_{2}\right)
\end{array}\right) \\
& T^{\mathrm{Y}}\left(L_{1}\right) \geq T^{\mathrm{Y}}\left(L_{2}\right) \vee \\
& \left(\begin{array}{c}
\left(T^{\mathrm{DUR}}\left(L_{1}\right)-T^{\mathrm{DUR}^{\mathrm{F}}}\left(L_{1}\right)\right) \cdot g \geq T_{2-1}^{\mathrm{Y}}+T_{2-1}^{\mathrm{YAR}} \wedge \\
T^{\mathrm{COST}}\left(L_{1}\right)+\rho^{\mathrm{TW}} \cdot T^{\mathrm{TW}}\left(L_{1}\right)+\psi\left(T_{2-1}^{\mathrm{Y}}+T_{2-1}^{\mathrm{YAR}}\right) \leq T^{\mathrm{COST}}\left(L_{2}\right)+\rho^{\mathrm{TW}} \cdot T^{\mathrm{TW}}\left(L_{2}\right)
\end{array}\right)
\end{aligned}
$$

where

$$
\begin{aligned}
T^{\mathrm{YAR}}\left(L_{i}\right)^{\prime} & =\min \left\{T^{\mathrm{YAR}}\left(L_{i}\right), T^{\mathrm{TAR}}\left(L_{i}\right) \cdot g\right\} \\
T_{i-j}^{\mathrm{RES}} & =T^{\mathrm{RES}}\left(L_{i}\right)-T^{\mathrm{RES}}\left(L_{j}\right) \\
\psi(y) & =y / c_{\mathrm{E}} \cdot c_{\mathrm{F}}
\end{aligned}
$$

Equations (A.29) and (A.30) are the same as before, comparing the penalized cost and time without electric energy. Equation (A.32) extends and replaces Equation (A.25) by taking into account the possibility of 
avoiding recharging times by using the ICE. The first part, as before, checks whether the duration of $L_{1}$ is lower than $L_{2}$. If it is not, a second check tests whether enough time can be reversed; if so, label $L_{1}$ has a still lower cost after the update. Equations (A.33) and (A.34) are defined similarly. If the total amount of electric energy available (or current electric energy available, respectively) of $L_{1}$ is less than $L_{2}$, then again the reversible amount is checked. If it is possible to reverse enough energy usage, the cost is again compared with the additional cost of using fossil fuel instead of electric energy.

\section{Appendix A.3. Detailed results for the E-FSMF}

Tables A.1, A.2 and A.3 show the results for the E-FSMF instance set. For the ALNS of Hiermann et al. (2016) (HPRH), the parallel matheuristic of Montoya (2016), and our HGA, we report the average, and the best objective value over 10 runs, as well as the average CPU time in minutes. The best known solution (BKS) prior to this work is shown in the column on the right side. The value at the bottom (Gap) presents the average deviation from the best known solution (i.e., Gap $\left.=\left(\mathrm{obj}-\mathrm{obj}^{\mathrm{BKS}}\right) / \mathrm{obj}^{\mathrm{BKS}}\right)$. 


\begin{tabular}{|c|c|c|c|c|c|c|c|c|c|c|}
\hline \multirow{2}{*}{$\begin{array}{l}\text { A } \\
\text { Name }\end{array}$} & \multicolumn{3}{|c|}{ HPRH } & \multicolumn{3}{|c|}{ Montoya } & \multicolumn{3}{|c|}{ HGA } & \multirow[t]{2}{*}{ BKS } \\
\hline & Avg 10 & Best 10 & $\mathrm{~T}(\min )$ & Avg 10 & Best 10 & $\mathrm{~T}(\min )$ & Avg 10 & Best 10 & $\mathrm{~T}(\min )$ & \\
\hline c101 & 7190.21 & 7180.42 & 17.53 & 7165.15 & 7162.01 & 25.88 & $\underline{7162.29}$ & $\underline{7160.77}$ & 21.12 & 7162.01 \\
\hline c102 & 7162.24 & 7154.50 & 17.95 & 7142.80 & 7139.45 & 26.02 & $\overline{7141.25}$ & $\overline{7137.04}$ & 21.86 & 7139.45 \\
\hline c103 & 7149.31 & 7126.29 & 18.30 & 7124.26 & 7121.12 & 31.22 & $\overline{7122.27}$ & $\overline{7117.32}$ & 22.93 & 7121.12 \\
\hline c104 & 7110.43 & 7100.22 & 17.75 & 7103.79 & 7099.88 & 32.93 & $\overline{7098.38}$ & $\overline{7097.80}$ & 23.99 & 7099.88 \\
\hline c105 & 7182.56 & 7155.23 & 17.60 & 7152.21 & 7140.31 & 27.24 & $\overline{7156.31}$ & 7138.85 & 21.15 & 7140.31 \\
\hline c106 & 7168.94 & 7146.88 & 17.69 & 7141.66 & 7136.90 & 27.28 & $\underline{7140.15}$ & $\overline{7134.75}$ & 21.92 & 7136.90 \\
\hline c107 & 7171.04 & 7156.18 & 17.42 & 7145.54 & 7139.12 & 30.49 & $\overline{7142.77}$ & $\overline{7136.44}$ & 21.24 & 7139.12 \\
\hline c108 & 7153.77 & 7141.49 & 17.53 & 7142.39 & 7133.30 & 30.22 & $\overline{7139.50}$ & $\overline{7131.83}$ & 21.99 & 7133.30 \\
\hline c109 & 7132.19 & 7120.33 & 17.32 & 7132.66 & 7123.15 & 33.32 & $\overline{7120.07}$ & $\overline{7113.94}$ & 22.39 & 7120.33 \\
\hline c201 & 5757.53 & 5737.57 & 39.28 & 5741.28 & 5736.35 & 19.36 & $\overline{5738.23}$ & $\overline{5736.11}$ & 13.72 & 5736.35 \\
\hline c202 & 5765.52 & 5744.65 & 42.22 & 5753.56 & 5740.43 & 21.50 & $\overline{5735.61}$ & $\overline{5733.53}$ & 15.76 & 5740.43 \\
\hline c203 & 5751.99 & 5726.08 & 47.69 & 5783.33 & 5744.25 & 28.27 & $\overline{5713.38}$ & $\overline{5713.38}$ & 18.73 & 5726.08 \\
\hline c204 & 5727.18 & 5705.82 & 50.91 & 5772.72 & 5724.75 & 41.49 & $\overline{5691.37}$ & $\overline{5689.04}$ & 21.21 & 5705.82 \\
\hline c205 & 5725.41 & 5703.48 & 46.16 & 5701.83 & 5694.58 & 24.43 & 5696.48 & 5693.45 & 15.21 & 5694.58 \\
\hline c206 & 5714.39 & 5708.77 & 31.20 & 5696.99 & 5689.08 & 26.40 & 5687.96 & 5687.96 & 16.90 & 5689.08 \\
\hline c207 & 5713.44 & 5697.99 & 32.58 & 5716.37 & 5696.54 & 27.61 & $\overline{5694.47}$ & $\overline{5693.23}$ & 17.30 & 5696.54 \\
\hline c208 & 5707.65 & 5685.40 & 50.23 & 5700.58 & 5682.60 & 30.48 & $\overline{5681.47}$ & $\overline{5681.47}$ & 16.10 & 5682.60 \\
\hline r101 & 4465.51 & 4426.85 & 14.47 & 4379.64 & 4366.21 & 21.41 & $\overline{4382.32}$ & $\overline{4372.29}$ & 9.12 & 4366.21 \\
\hline r102 & 4270.92 & 4245.82 & 14.97 & 4180.61 & 4176.82 & 13.11 & 4202.05 & 4186.74 & 10.06 & 4176.82 \\
\hline r103 & 4130.86 & 4103.35 & 16.43 & 4049.01 & 4043.29 & 15.53 & 4060.29 & 4048.37 & 11.82 & 4043.29 \\
\hline r104 & 4025.60 & 4007.28 & 15.27 & 3970.77 & 3969.12 & 21.45 & 3972.91 & 3966.74 & 13.77 & 3969.12 \\
\hline r105 & 4215.34 & 4181.80 & 15.37 & 4140.08 & 4138.01 & 12.94 & 4154.04 & $\overline{4145.27}$ & 9.51 & 4138.01 \\
\hline r106 & 4155.24 & 4120.23 & 15.54 & 4075.44 & 4068.15 & 16.46 & 4087.59 & 4078.17 & 11.22 & 4068.15 \\
\hline r107 & 4093.59 & 4057.06 & 15.30 & 4008.71 & 4003.84 & 24.07 & 4011.87 & $\underline{4000.69}$ & 12.26 & 4003.84 \\
\hline r108 & 4025.75 & 3992.57 & 15.77 & 3961.93 & 3955.48 & 23.96 & 3968.72 & $\overline{3961.92}$ & 17.29 & 3955.48 \\
\hline r109 & 4110.98 & 4067.14 & 15.58 & 4024.49 & 4016.33 & 19.92 & 4034.36 & 4023.64 & 11.85 & 4016.33 \\
\hline r110 & 4045.96 & 4024.71 & 15.73 & 3975.95 & 3969.02 & 26.51 & 3991.65 & 3973.72 & 16.88 & 3969.02 \\
\hline r111 & 4048.42 & 4023.38 & 15.93 & 3976.91 & 3972.84 & 25.10 & 3990.36 & 3984.77 & 16.66 & 3972.84 \\
\hline r112 & 4023.01 & 4001.87 & 15.80 & 3947.93 & 3942.68 & 21.85 & 3955.19 & $\underline{3942.66}$ & 16.23 & 3942.68 \\
\hline r201 & 3432.83 & 3413.93 & 42.20 & 3543.81 & 3515.45 & 17.91 & 3410.53 & 3399.82 & 17.61 & 3413.93 \\
\hline r202 & 3295.26 & 3270.49 & 44.95 & 3397.33 & 3366.13 & 18.23 & 3275.73 & 3266.47 & 20.93 & 3270.49 \\
\hline r203 & 3169.97 & 3136.47 & 49.40 & 3252.78 & 3224.34 & 23.86 & 3137.34 & 3127.56 & 24.10 & 3136.47 \\
\hline r204 & 3026.09 & 3008.01 & 46.32 & 3114.40 & 3098.94 & 26.84 & $\overline{3003.15}$ & $\overline{3002.72}$ & 29.11 & 3008.01 \\
\hline r205 & 3261.16 & 3234.26 & 40.89 & 3356.01 & 3326.29 & 21.28 & $\overline{3240.34}$ & $\overline{3230.20}$ & 20.48 & 3234.26 \\
\hline r206 & 3194.12 & 3172.50 & 47.73 & 3296.06 & 3261.05 & 26.25 & $\overline{3161.18}$ & $\overline{3156.58}$ & 22.96 & 3172.50 \\
\hline r207 & 3099.52 & 3079.39 & 46.87 & 3175.13 & 3159.76 & 23.25 & $\overline{3062.33}$ & 3059.85 & 28.94 & 3079.39 \\
\hline r208 & 3026.57 & 3010.51 & 51.26 & 3104.77 & 3088.29 & 33.89 & $\overline{2999.40}$ & $\overline{2995.96}$ & 34.17 & 3010.51 \\
\hline r209 & 3161.57 & 3142.72 & 45.06 & 3255.62 & 3224.28 & 25.29 & 3133.33 & 3122.41 & 23.40 & 3142.72 \\
\hline r210 & 3143.79 & 3110.90 & 45.94 & 3235.09 & 3181.95 & 25.02 & 3109.03 & 3101.10 & 22.95 & 3110.90 \\
\hline $\mathrm{r} 211$ & 3079.24 & 3041.93 & 44.29 & 3147.32 & 3133.26 & 20.83 & 3030.99 & 3026.74 & 28.18 & 3041.93 \\
\hline rc101 & 5346.49 & 5294.01 & 14.13 & 5255.03 & 5247.39 & 13.21 & $\overline{5261.36}$ & $\overline{5254.50}$ & 9.06 & 5247.39 \\
\hline $\mathrm{rc} 102$ & 5180.03 & 5121.53 & 14.63 & 5118.29 & 5114.15 & 24.81 & 5096.49 & 5069.43 & 10.34 & 5114.15 \\
\hline rc103 & 5007.37 & 4958.51 & 14.53 & 4924.38 & 4916.98 & 18.60 & & 4905.29 & 9.41 & 4916.98 \\
\hline $\mathrm{rc} 104$ & 4862.65 & 4804.00 & 16.03 & 4815.22 & 4801.06 & 24.28 & 4808.85 & 4783.16 & 10.50 & 4801.06 \\
\hline rc105 & 5117.09 & 5074.43 & 14.48 & 5068.85 & 5060.96 & 14.74 & $\overline{5063.17}$ & $\overline{5044.93}$ & 9.95 & 5060.96 \\
\hline rc106 & 5102.46 & 5028.28 & 14.69 & 5001.29 & 4985.03 & 14.12 & $\overline{5002.07}$ & $\overline{4991.29}$ & 9.97 & 4985.03 \\
\hline $\mathrm{rc} 107$ & 4913.90 & 4864.78 & 15.24 & 4853.96 & 4835.75 & 15.67 & 4859.83 & 4836.81 & 10.66 & 4835.75 \\
\hline $\mathrm{rc} 108$ & 4862.41 & 4814.33 & 15.64 & 4819.12 & 4798.48 & 23.59 & $\underline{4815.83}$ & 4800.17 & 10.25 & 4798.48 \\
\hline rc201 & 4361.17 & 4346.25 & 14.27 & 4388.86 & 4371.85 & 32.88 & 4343.47 & 4337.60 & 21.58 & 4346.25 \\
\hline rc202 & 4295.27 & 4273.74 & 14.59 & 4313.51 & 4288.62 & 31.02 & $\underline{4271.96}$ & 4267.75 & 24.23 & 4273.74 \\
\hline rc203 & 4186.28 & 4152.94 & 15.98 & 4227.27 & 4191.76 & 36.69 & $\overline{4152.79}$ & $\underline{4147.68}$ & 26.63 & 4152.94 \\
\hline rc204 & 4127.11 & 4113.49 & 19.18 & 4160.55 & 4136.86 & 46.62 & $\overline{4096.59}$ & $\overline{4094.11}$ & 29.46 & 4113.49 \\
\hline rc205 & 4273.59 & 4246.52 & 14.86 & 4294.99 & 4274.33 & 33.33 & $\overline{4248.30}$ & $\overline{4242.63}$ & 23.58 & 4246.52 \\
\hline rc206 & 4270.25 & 4237.75 & 15.09 & 4289.85 & 4260.22 & 33.95 & 4241.71 & 4236.43 & 24.08 & 4237.75 \\
\hline rc207 & 4199.60 & 4177.23 & 16.20 & 4242.41 & 4215.09 & 36.42 & $\overline{4174.39}$ & $\overline{4169.59}$ & 26.14 & 4177.23 \\
\hline rc208 & 4122.12 & 4097.04 & 18.13 & 4173.32 & 4142.05 & 42.75 & 4103.79 & 4096.01 & 30.99 & 4097.04 \\
\hline $\begin{array}{l}\text { Avg } \\
\text { Gap }\end{array}$ & $0.94 \%$ & $0.36 \%$ & 25.68 & $0.80 \%$ & $0.46 \%$ & 5.57 & $0.07 \%$ & $-0.10 \%$ & 18.75 & \\
\hline
\end{tabular}

Table A.1: Comparison with the best known solutions for the E-FSMFTW, Type-A. BnP/HPRH: Hiermann et al. (2016); Montoya (2016); The best values are indicated in boldface (also underlined for new best values). 


\begin{tabular}{|c|c|c|c|c|c|c|c|c|c|c|}
\hline \multirow{2}{*}{$\begin{array}{l}\text { B } \\
\text { Name }\end{array}$} & \multicolumn{3}{|c|}{ HPRH } & \multicolumn{3}{|c|}{ Montoya } & \multicolumn{3}{|c|}{ HGA } & \multirow[t]{2}{*}{ BKS } \\
\hline & Avg 10 & Best 10 & $\mathrm{~T}(\min )$ & Avg 10 & Best 10 & $\mathrm{~T}(\min )$ & Avg 10 & Best 10 & $\mathrm{~T}(\min )$ & \\
\hline c101 & 2505.73 & 2495.00 & 14.43 & 2502.09 & 2495.00 & 25.56 & $\underline{2496.28}$ & 2495.00 & 13.59 & 2495.00 \\
\hline c102 & 2450.73 & 2445.99 & 14.41 & 2451.39 & 2448.76 & 25.86 & $\overline{2446.48}$ & 2445.99 & 13.85 & 2445.99 \\
\hline c103 & 2452.40 & 2438.54 & 15.03 & 2437.99 & 2430.72 & 31.16 & $\overline{2435.42}$ & $\underline{2427.44}$ & 19.49 & 2430.72 \\
\hline c104 & 2428.95 & 2404.97 & 15.07 & 2410.12 & 2404.73 & 32.85 & $\overline{2404.40}$ & $\overline{2402.47}$ & 20.12 & 2404.73 \\
\hline c105 & 2475.95 & 2472.93 & 14.45 & 2476.46 & 2473.79 & 27.16 & $\overline{2473.14}$ & $\overline{2472.60}$ & 17.21 & 2472.93 \\
\hline c106 & 2468.13 & 2462.54 & 14.71 & 2466.25 & 2462.90 & 27.20 & $\overline{2459.90}$ & $\overline{2456.74}$ & 16.63 & 2462.54 \\
\hline c107 & 2461.32 & 2458.37 & 14.60 & 2459.31 & 2458.37 & 30.25 & $\overline{2457.98}$ & $\overline{2457.89}$ & 16.53 & 2458.37 \\
\hline c108 & 2463.02 & 2450.17 & 14.33 & 2455.74 & 2452.58 & 30.17 & $\overline{2451.13}$ & $\overline{2448.08}$ & 18.82 & 2450.17 \\
\hline c109 & 2452.57 & 2436.41 & 15.07 & 2434.02 & 2431.87 & 33.22 & $\overline{2432.77}$ & $\overline{2431.36}$ & 19.22 & 2431.87 \\
\hline c201 & 1739.26 & 1730.41 & 35.76 & 1730.50 & 1730.41 & 14.14 & $\overline{1730.41}$ & $\overline{1730.41}$ & 14.51 & 1730.41 \\
\hline c202 & 1745.24 & 1737.57 & 38.14 & 1730.12 & 1729.73 & 17.01 & $\overline{1730.05}$ & 1729.73 & 16.40 & 1729.73 \\
\hline c203 & 1742.76 & 1716.29 & 39.38 & 1743.23 & 1730.26 & 23.73 & $\overline{1713.38}$ & 1713.38 & 18.46 & 1716.29 \\
\hline c204 & 1709.43 & 1699.07 & 37.02 & 1753.55 & 1724.75 & 36.43 & $\overline{1690.01}$ & $\overline{1689.04}$ & 21.42 & 1699.07 \\
\hline c205 & 1715.09 & 1697.01 & 37.83 & 1701.83 & 1694.58 & 17.33 & $\overline{1694.83}$ & $\overline{1693.45}$ & 15.38 & 1694.58 \\
\hline c206 & 1712.38 & 1693.15 & 36.78 & 1696.99 & 1689.08 & 20.69 & $\overline{1687.96}$ & $\overline{1687.96}$ & 16.68 & 1689.08 \\
\hline c207 & 1710.70 & 1694.61 & 35.05 & 1715.61 & 1696.54 & 22.19 & $\overline{1694.47}$ & 1693.23 & 16.92 & 1694.61 \\
\hline c208 & 1707.11 & 1681.47 & 36.46 & 1701.29 & 1682.60 & 24.49 & 1681.47 & $\overline{1681.47}$ & 16.56 & 1681.47 \\
\hline r101 & 2281.28 & 2261.21 & 13.68 & 2253.93 & 2253.04 & 10.71 & $\overline{2258.24}$ & 2249.24 & 7.75 & 2249.14 \\
\hline r102 & 2095.87 & 2073.03 & 14.45 & 2057.97 & 2053.42 & 8.01 & 2063.58 & 2047.89 & 8.80 & 2047.89 \\
\hline r103 & 1927.52 & 1894.98 & 15.11 & 1897.06 & 1892.84 & 13.13 & 1906.46 & 1898.26 & 9.50 & 1892.84 \\
\hline r104 & 1775.33 & 1747.65 & 15.28 & 1761.84 & 1749.48 & 22.78 & 1766.32 & 1754.22 & 10.63 & 1747.65 \\
\hline r105 & 2030.12 & 2010.31 & 15.08 & 2007.01 & 1998.80 & 9.17 & 2013.63 & 2007.40 & 8.79 & 1997.75 \\
\hline r106 & 1963.88 & 1934.00 & 14.51 & 1929.13 & 1925.83 & 12.02 & 1948.02 & 1925.56 & 9.52 & 1925.83 \\
\hline r107 & 1844.20 & 1824.88 & 15.50 & 1820.75 & 1818.03 & 22.10 & 1842.49 & $\overline{1824.68}$ & 10.21 & 1818.03 \\
\hline r108 & 1753.09 & 1729.18 & 16.27 & 1733.87 & 1720.78 & 23.31 & 1732.21 & 1712.40 & 10.93 & 1720.78 \\
\hline r109 & 1904.12 & 1871.54 & 15.37 & 1867.32 & 1863.48 & 25.36 & 1877.84 & 1861.15 & 9.75 & 1863.48 \\
\hline r110 & 1793.48 & 1759.69 & 15.54 & 1755.92 & 1752.40 & 12.89 & 1786.94 & $\overline{1766.10}$ & 10.67 & 1752.40 \\
\hline r111 & 1808.36 & 1786.97 & 15.66 & 1775.04 & 1765.08 & 22.45 & 1793.86 & 1769.41 & 10.62 & 1765.08 \\
\hline r112 & 1746.02 & 1721.79 & 15.77 & 1722.34 & 1714.93 & 26.75 & $\underline{1717.98}$ & $\underline{1705.89}$ & 10.74 & 1714.93 \\
\hline r201 & 1618.25 & 1594.58 & 31.21 & 1714.30 & 1697.28 & 40.11 & $\overline{1597.16}$ & 1591.35 & 14.57 & 1594.58 \\
\hline r202 & 1479.42 & 1468.05 & 29.61 & 1551.73 & 1541.45 & 17.87 & 1468.59 & 1461.63 & 18.31 & 1468.05 \\
\hline r203 & 1354.24 & 1340.00 & 30.70 & 1438.89 & 1424.34 & 23.68 & 1334.67 & 1327.56 & 20.54 & 1340.00 \\
\hline r204 & 1211.63 & 1203.89 & 27.35 & 1286.75 & 1278.59 & 27.33 & $\overline{1205.19}$ & $\overline{1202.72}$ & 26.38 & 1203.89 \\
\hline r205 & 1455.08 & 1430.70 & 30.16 & 1534.24 & 1524.53 & 20.82 & $\overline{1433.53}$ & $\overline{1429.32}$ & 17.53 & 1430.70 \\
\hline r206 & 1376.34 & 1361.69 & 31.35 & 1470.36 & 1461.05 & 27.07 & $\overline{1357.28}$ & $\overline{1355.90}$ & 19.56 & 1361.69 \\
\hline r207 & 1268.66 & 1256.22 & 28.18 & 1364.12 & 1359.76 & 23.60 & $\overline{1260.31}$ & $\overline{1257.88}$ & 24.61 & 1256.22 \\
\hline r208 & 1208.89 & 1198.39 & 29.02 & 1289.03 & 1281.92 & 34.43 & $\overline{1198.80}$ & 1195.96 & 28.35 & 1198.39 \\
\hline r209 & 1345.50 & 1333.33 & 30.30 & 1407.39 & 1381.34 & 25.18 & $\overline{1329.72}$ & $\overline{1321.76}$ & 20.01 & 1333.33 \\
\hline r210 & 1324.07 & 1314.16 & 30.07 & 1385.87 & 1359.10 & 25.44 & 1301.00 & 1298.78 & 19.12 & 1314.16 \\
\hline $\mathrm{r} 211$ & 1244.73 & 1231.38 & 26.30 & 1326.35 & 1319.15 & 21.33 & 1233.22 & 1226.74 & 23.49 & 1231.38 \\
\hline rc101 & 2560.33 & 2548.84 & 13.71 & 2508.65 & 2504.72 & 9.42 & 2514.33 & 2501.78 & 8.63 & 2504.72 \\
\hline rc102 & 2359.92 & 2330.50 & 14.35 & 2335.72 & 2334.08 & 9.68 & 2326.17 & 2315.01 & 9.07 & 2330.50 \\
\hline rc103 & 2136.78 & 2105.84 & 14.14 & 2113.31 & 2108.82 & 10.78 & 2126.76 & 2116.31 & 9.79 & 2105.84 \\
\hline rc104 & 2002.33 & 1986.35 & 15.56 & 1984.66 & 1979.16 & 14.39 & 1980.44 & 1973.78 & 10.48 & 1979.16 \\
\hline rc105 & 2287.95 & 2259.97 & 13.87 & 2264.49 & 2252.05 & 9.81 & $\overline{2259.34}$ & $\overline{2250.37}$ & 9.18 & 2252.05 \\
\hline rc106 & 2232.05 & 2209.73 & 14.50 & 2194.98 & 2187.30 & 10.21 & $\overline{2213.35}$ & $\overline{2194.61}$ & 9.71 & 2187.30 \\
\hline rc107 & 2050.20 & 2037.25 & 15.43 & 2039.77 & 2034.35 & 15.20 & 2043.64 & 2012.53 & 9.69 & 2034.35 \\
\hline rc108 & 1995.41 & 1962.87 & 14.99 & 1975.91 & 1969.75 & 16.03 & 1974.87 & 1967.99 & 9.98 & 1962.87 \\
\hline rc201 & 1931.42 & 1899.99 & 15.69 & 2013.94 & 1976.91 & 16.64 & 1912.42 & 1899.99 & 10.71 & 1899.99 \\
\hline rc202 & 1825.07 & 1807.30 & 16.19 & 1921.19 & 1876.72 & 19.55 & $\overline{1807.50}$ & 1805.24 & 14.68 & 1807.30 \\
\hline rc203 & 1660.93 & 1642.43 & 19.00 & 1749.83 & 1717.98 & 22.51 & $\overline{1649.11}$ & $\overline{1637.32}$ & 15.48 & 1642.43 \\
\hline rc204 & 1543.04 & 1521.80 & 22.13 & 1638.89 & 1597.07 & 34.12 & $\overline{1526.04}$ & $\overline{1520.59}$ & 19.91 & 1521.80 \\
\hline rc205 & 1774.22 & 1753.79 & 19.03 & 1892.25 & 1832.28 & 21.98 & $\overline{1758.96}$ & $\overline{1747.39}$ & 13.08 & 1753.79 \\
\hline rc206 & 1767.75 & 1751.75 & 17.79 & 1883.33 & 1819.18 & 23.71 & 1757.36 & 1742.98 & 13.91 & 1751.75 \\
\hline rc207 & 1640.23 & 1616.96 & 19.07 & 1782.89 & 1694.69 & 27.96 & 1611.17 & 1603.23 & 15.34 & 1616.96 \\
\hline rc208 & 1520.76 & 1497.95 & 22.03 & 1660.49 & 1578.86 & 32.61 & 1499.75 & 1495.34 & 21.06 & 1497.95 \\
\hline $\begin{array}{l}\text { Avg } \\
\text { Gap }\end{array}$ & $1.23 \%$ & $0.22 \%$ & 47 & $2.33 \%$ & $1.52 \%$ & 96 & $0.29 \%$ & $-0.13 \%$ & 15.05 & \\
\hline
\end{tabular}

Table A.2: Comparison with the best known solutions for the E-FSMFTW, Type-B. HPRH: Hiermann et al. (2016); Montoya (2016); The best values are indicated in boldface (also underlined for new best values). 


\begin{tabular}{|c|c|c|c|c|c|c|c|c|c|c|}
\hline \multirow{2}{*}{$\begin{array}{l}\text { C } \\
\text { Name }\end{array}$} & \multicolumn{3}{|c|}{ HPRH } & \multicolumn{3}{|c|}{ Montoya } & \multicolumn{3}{|c|}{ HGA } & \multirow[t]{2}{*}{ BKS } \\
\hline & Avg 10 & Best 10 & $\mathrm{~T}(\min )$ & Avg 10 & Best 10 & $\mathrm{~T}(\min )$ & Avg 10 & Best 10 & $\mathrm{~T}(\min )$ & \\
\hline c101 & 1816.06 & 1810.12 & 14.08 & 1820.45 & 1810.12 & 8.84 & 1809.97 & 1809.93 & 7.06 & $1809.93^{*}$ \\
\hline c102 & 1766.14 & 1759.73 & 14.36 & 1770.44 & 1763.97 & 9.20 & $\mathbf{1 7 5 9 . 7 3}$ & 1759.73 & 7.91 & 1759.73 \\
\hline c103 & 1759.20 & 1755.02 & 15.09 & 1757.68 & 1746.80 & 20.76 & 1745.33 & 1744.92 & 11.06 & 1746.80 \\
\hline c104 & 1735.86 & 1719.67 & 15.60 & 1727.72 & 1721.53 & 29.04 & $\overline{1718.73}$ & $\overline{1717.33}$ & 10.10 & 1719.67 \\
\hline c105 & 1785.43 & 1783.25 & 14.51 & 1790.59 & 1785.31 & 10.66 & $\overline{1783.92}$ & $\overline{1783.25}$ & 7.19 & 1783.25 \\
\hline c106 & 1777.67 & 1774.77 & 14.64 & 1780.39 & 1775.08 & 11.10 & $\overline{1773.57}$ & 1772.74 & 8.09 & 1774.77 \\
\hline c107 & 1768.33 & 1764.02 & 14.57 & 1775.74 & 1765.45 & 14.68 & 1765.09 & 1764.02 & 7.90 & 1764.02 \\
\hline c108 & 1769.76 & 1761.41 & 14.80 & 1772.78 & 1768.05 & 20.58 & $\overline{1762.95}$ & $\underline{1760.63}$ & 8.79 & 1761.41 \\
\hline c109 & 1749.07 & 1740.18 & 15.30 & 1749.05 & 1744.09 & 29.92 & $\overline{1740.78}$ & $\overline{1738.93}$ & 9.17 & 1740.18 \\
\hline c201 & 1213.63 & 1210.41 & 31.30 & 1210.50 & 1210.41 & 13.50 & $\overline{1210.41}$ & $\overline{1210.41}$ & 13.84 & 1210.41 \\
\hline c202 & 1220.97 & 1209.73 & 34.17 & 1210.12 & 1209.73 & 16.58 & 1209.86 & 1209.73 & 15.72 & 1209.73 \\
\hline c203 & 1227.69 & 1212.34 & 33.07 & 1226.22 & 1216.18 & 30.01 & 1208.22 & 1207.95 & 17.96 & 1212.34 \\
\hline c204 & 1199.37 & 1179.25 & 32.15 & 1232.00 & 1204.68 & 35.62 & $\overline{1177.50}$ & $\overline{1175.94}$ & 20.00 & 1179.25 \\
\hline c205 & 1195.24 & 1188.92 & 31.64 & 1195.35 & 1192.54 & 16.70 & 1192.69 & $\overline{1188.92}$ & 15.94 & 1188.92 \\
\hline c206 & 1192.30 & 1183.42 & 31.01 & 1191.74 & 1188.81 & 20.16 & 1183.42 & 1183.42 & 17.40 & 1183.42 \\
\hline $\mathrm{c} 207$ & 1190.37 & 1183.42 & 31.44 & 1190.65 & 1187.49 & 21.52 & 1183.42 & 1183.42 & 17.17 & 1183.42 \\
\hline c208 & 1192.96 & 1181.47 & 30.82 & 1192.42 & 1182.60 & 23.78 & 1181.47 & 1181.47 & 16.70 & 1181.47 \\
\hline r101 & 1977.89 & 1961.02 & 14.36 & 1959.07 & 1959.04 & 9.45 & 1957.89 & 1954.56 & 7.82 & $1954.00^{*}$ \\
\hline r102 & 1791.03 & 1765.36 & 14.67 & 1767.00 & 1761.48 & 7.54 & $\mathbf{1 7 6 0 . 9 2}$ & 1757.91 & 8.64 & $1757.13^{*}$ \\
\hline r103 & 1618.81 & 1601.23 & 15.52 & 1593.13 & 1587.32 & 12.54 & $\overline{1603.05}$ & 1589.17 & 9.65 & 1587.32 \\
\hline r104 & 1448.31 & 1424.30 & 16.20 & 1437.37 & 1427.76 & 19.66 & 1435.56 & 1417.69 & 10.97 & 1424.30 \\
\hline r105 & 1728.12 & 1704.36 & 14.68 & 1705.56 & 1699.34 & 8.25 & $\overline{1714.26}$ & $\overline{1708.92}$ & 9.10 & $1699.34^{*}$ \\
\hline r106 & 1635.42 & 1611.62 & 14.67 & 1612.20 & 1609.78 & 8.55 & 1623.31 & 1603.24 & 9.55 & 1604.55 \\
\hline r107 & 1514.01 & 1490.04 & 15.99 & 1499.00 & 1493.12 & 17.35 & 1500.84 & 1493.27 & 10.27 & 1490.04 \\
\hline r108 & 1417.39 & 1399.27 & 16.42 & 1404.05 & 1397.86 & 18.13 & 1399.22 & 1389.46 & 10.58 & 1397.86 \\
\hline r109 & 1580.14 & 1560.34 & 15.65 & 1554.22 & 1551.69 & 16.98 & 1566.61 & 1552.48 & 10.03 & 1550.40 \\
\hline r110 & 1471.66 & 1446.48 & 15.64 & 1429.38 & 1420.13 & 12.11 & 1447.59 & 1434.84 & 10.43 & 1420.13 \\
\hline r111 & 1479.75 & 1457.68 & 16.16 & 1443.94 & 1439.02 & 12.62 & 1460.14 & 1441.99 & 10.53 & 1438.81 \\
\hline r112 & 1403.82 & 1389.87 & 16.10 & 1394.19 & 1388.22 & 25.74 & 1398.31 & 1385.84 & 10.98 & 1388.22 \\
\hline r201 & 1378.77 & 1366.63 & 29.69 & 1480.55 & 1474.75 & 39.50 & 1378.37 & 1368.94 & 13.30 & 1366.63 \\
\hline r202 & 1249.65 & 1236.97 & 29.13 & 1328.35 & 1316.45 & 42.06 & 1249.56 & 1245.49 & 17.10 & 1236.97 \\
\hline r203 & 1124.07 & 1104.85 & 30.23 & 1205.32 & 1183.71 & 56.20 & 1107.65 & 1102.56 & 19.49 & 1104.85 \\
\hline r204 & 983.97 & 977.72 & 26.81 & 1050.51 & 1032.48 & 65.96 & 978.93 & 977.72 & 24.84 & 977.72 \\
\hline $\mathrm{r} 205$ & 1232.63 & 1217.77 & 29.15 & 1308.90 & 1293.54 & 47.77 & 1205.62 & 1197.20 & 17.35 & 1217.77 \\
\hline r206 & 1155.47 & 1136.83 & 30.95 & 1239.18 & 1230.67 & 60.69 & 1133.39 & 1130.90 & 18.26 & 1136.83 \\
\hline r 207 & 1057.22 & 1031.22 & 26.31 & 1136.77 & 1110.93 & 58.58 & $\overline{1033.95}$ & $\overline{1031.22}$ & 23.80 & 1031.22 \\
\hline r208 & 984.87 & 971.15 & 28.21 & 1049.20 & 1032.20 & 85.72 & $\overline{973.34}$ & $\underline{970.96}$ & 28.08 & 971.15 \\
\hline r209 & 1117.68 & 1099.24 & 29.62 & 1170.58 & 1156.34 & 57.01 & 1100.36 & 1092.26 & 18.11 & 1099.24 \\
\hline $\mathrm{r} 210$ & 1100.27 & 1087.21 & 29.88 & 1143.99 & 1134.10 & 57.33 & $\underline{1077.86}$ & $\underline{1069.71}$ & 18.35 & 1087.21 \\
\hline $\mathrm{r} 211$ & 1026.07 & 1006.38 & 25.93 & 1098.37 & 1087.95 & 51.81 & 1005.16 & 1001.74 & 22.23 & 1006.38 \\
\hline rc101 & 2153.24 & 2142.24 & 13.80 & 2123.26 & 2121.02 & 8.96 & $\overline{2134.84}$ & 2119.70 & 8.96 & 2121.02 \\
\hline rc102 & 1972.85 & 1957.11 & 14.68 & 1950.16 & 1947.54 & 9.40 & 1959.96 & 1945.31 & 9.60 & 1947.54 \\
\hline rc103 & 1764.22 & 1736.25 & 14.54 & 1736.49 & 1726.85 & 10.72 & 1745.83 & 1733.70 & 10.03 & 1726.85 \\
\hline rc104 & 1614.09 & 1595.44 & 15.80 & 1605.91 & 1596.18 & 15.20 & 1596.48 & 1584.79 & 10.40 & 1595.44 \\
\hline rc105 & 1900.42 & 1885.63 & 14.14 & 1880.03 & 1875.91 & 9.93 & 1881.65 & 1870.80 & 9.67 & 1875.91 \\
\hline rc106 & 1844.99 & 1823.89 & 15.18 & 1814.49 & 1811.86 & 9.96 & 1814.28 & 1808.96 & 9.90 & 1811.86 \\
\hline rc107 & 1675.58 & 1639.84 & 15.31 & 1659.27 & 1645.79 & 15.60 & 1656.95 & 1635.51 & 10.19 & 1639.84 \\
\hline rc108 & 1601.47 & 1578.51 & 14.98 & 1588.87 & 1583.70 & 14.15 & 1586.20 & 1583.08 & 10.91 & 1578.51 \\
\hline rc201 & 1617.52 & 1589.99 & 15.64 & 1682.78 & 1664.90 & 14.79 & $\underline{\mathbf{1 5 9 8 . 6 9}}$ & 1588.25 & 11.07 & 1589.99 \\
\hline rc202 & 1497.99 & 1485.13 & 16.72 & 1587.09 & 1541.00 & 15.11 & 1483.05 & 1481.05 & 15.40 & 1485.13 \\
\hline rc203 & 1333.25 & 1310.37 & 19.35 & 1363.51 & 1345.08 & 48.90 & 1316.19 & 1310.48 & 16.57 & 1310.37 \\
\hline rc204 & 1193.93 & 1183.16 & 22.69 & 1224.22 & 1212.07 & 77.59 & 1186.42 & 1182.32 & 21.22 & 1183.16 \\
\hline rc205 & 1440.00 & 1424.75 & 20.95 & 1508.13 & 1500.24 & 39.75 & $\overline{1426.02}$ & $\overline{1422.39}$ & 14.55 & 1424.75 \\
\hline rc206 & 1439.17 & 1431.21 & 18.11 & 1493.94 & 1473.86 & 43.46 & 1434.12 & 1429.47 & 14.54 & 1431.21 \\
\hline rc207 & 1299.14 & 1277.71 & 21.30 & 1364.75 & 1344.75 & 49.95 & 1280.53 & 1273.23 & 15.47 & 1277.71 \\
\hline rc208 & 1171.52 & 1161.57 & 22.91 & 1216.16 & 1202.33 & 64.83 & 1163.21 & 1159.70 & 22.02 & 1161.57 \\
\hline $\begin{array}{l}\text { Avg } \\
\text { Gap }\end{array}$ & $1.27 \%$ & $0.21 \%$ & 20.83 & $2.31 \%$ & $1.64 \%$ & 28.08 & $0.33 \%$ & $-0.10 \%$ & 13.61 & \\
\hline
\end{tabular}

Table A.3: Comparison with the best known solutions (BKS) for the E-FSMFTW, Type-C. HPRH: Hiermann et al. (2016); Montoya (2016); The best values are indicated in boldface (also underlined for new best values). BKS marked with * are proven optimal by the branch-and-price algorithm described in Hiermann et al. (2016). 